\title{
Downscaling Images with Trends using Multiple-point Statistics Simulation: An Application to Digital Elevation Models
}

\author{
Luiz Gustavo Rasera · Mathieu Gravey · \\ Stuart N. Lane · Gregoire Mariethoz
}

Received: 31 January 2019 / Accepted: ???

\begin{abstract}
Remote sensing and geophysical imaging techniques are often limited in terms of spatial resolution. This prevents the characterization of physical properties and processes at scales finer than the spatial resolution provided by the imaging sensor. In the last decade, multiple-point statistics simulation has been successfully used for downscaling problems. In this approach, the missing fine scale structures are imported from a training image which describes the correspondence between coarse and equivalent fine scale structures. However, in many cases, large variations in the amplitude of the imaged physical attribute, known as trends, pose a challenge for the detection and simulation of these fine scale features. Here, we develop a novel multiple-point statistics simulation method for downscaling coarse resolution images with trends. The proposed algorithm relies on a multi-scale sequential simulation framework. Trends in the data are handled by an inbuilt decomposition of the target variable into a deterministic trend component and a stochastic residual component at multiple scales. We also introduce the application of kernel weighting for computing distances between data events and probability aggregation operations for integrating different support data based on a distance-to-probability transformation function. The algorithm is benchmarked against two-point and multiple-point statistics simulation methods, and a deterministic interpolation technique. Results show that the approach is able to cope with non-stationary data sets and scenarios in which the statistics of the training image differ from the conditioning data statistics. Two case studies using digital elevation models of mountain ranges in Switzerland illustrate the method.
\end{abstract}

L.G. Rasera ( $\varangle) \cdot$ M. Gravey $\cdot$ S.L. Lane $\cdot$ G. Mariethoz

Institute of Earth Surface Dynamics, Faculty of Geosciences and Environment, University of Lausanne, 1015 Lausanne, Switzerland

E-mail: luizgustavo.rasera@unil.ch 
Keywords Statistical downscaling · Trends · Multiple-point statistics · Simulation · Training image $\cdot$ Digital elevation model

\section{Introduction}

Surface and subsurface investigations often need to estimate phenomena at scales finer than the spatial resolution provided by imaging sensors. Downscaling methods are commonly employed to achieve this. Atkinson (2013) provides an overview of statistical downscaling in remote sensing. From a statistical perspective, downscaling is an ill-posed problem because the upscaling of different fine resolution images may result in the same coarse scale image (Bertero and Boccacci 1998 Boucher and Kyriakidis 2007). The problem is resolved by producing multiple equiprobable synthetic fine resolution images. This allows determination of the uncertainty associated with the sub-pixel predictions, and propagation of the impact of the fine scale uncertainty to the response of a target transfer function. Therefore, the goal is to produce a finer resolution version of the original image, which is coherent with its low-resolution counterpart, and a given prior fine scale structural model.

Geostatistical simulation provides a potential framework for stochastic downscaling problems. Traditional covariance-based simulation methods (Goovaerts 1997) have been adapted for downscaling and integration of coarse and fine scale data (Journel 1999 Kyriakidis and Yoo 2005 Boucher and Kyriakidis 2007 Liu and Journel 2009 Zagayevskiy and Deutsch 2015). Two-point simulation has also been applied for conflation and downscaling of terrain elevation data (Kyriakidis et al. $1999 \mathrm{Hengl}$ et al. 2008). These methods assume that the second-order statistics characterized through variogram models are sufficient for describing the missing fine scale structures. In addition, two-point statistics simulation approaches implicitly adopt the higher-order statistics embedded in the simulation algorithm (Remy et al. 2009). These higherorder statistics are often high-entropy in character, which leads to maximization of the spatial disorder beyond the input variogram model (Journel and Deutsch 1993). Such assumptions may be inappropriate for modeling low entropy Earth textures that depict spatial connectivity between extreme data values, such as permeability in porous media (Renard and Allard 2013), curvilinear geological structures (Strebelle 2002), and topographic features including surface drainage networks (Tang et al. 2015).

Multiple-point statistics (MPS) simulation (Remy et al. 2009) offers an alternative to two-point statistics simulation for modeling low entropy textures. It does not require the definition of an explicit random function model. Rather, the task of generating a simulated realization is formulated as a stochastic imaging problem. The structural model is commonly referred to as a training image, which consists of an analog or a conceptual representation of the studied phenomenon. The spatial structure and statistics of the random field are then extracted from the training image based on computed conditional probability distribution functions (CPDFs) (Guardiano and Srivastava 1993 Strebelle 2002), or by direct sampling (Mariethoz 
et al. 2010). Boucher (2009b), Mariethoz et al. (2011), and Straubhaar et al. (2016) proposed different applications of MPS simulation for downscaling problems.

Another common characteristic of Earth science data sets is the presence of trends. Trends consist of large scale variations, usually low spatial frequencies, of the physical property under study. In these cases, the expected values of the random variables (RVs) representing such properties are deemed unknown (Journel and Rossi 1989). These local expectations are often modeled with a trend function (e.g. a locally varying mean) that can be a function of the spatial coordinates of the regionalized variable, or an estimate of the expected value based on a correlated auxiliary variable. The RV is thus decomposed into two components: a deterministic low-frequency trend and its associated complementary stochastic higher-frequency residual. Tang et al. (2015) used a similar approach for digital elevation data fusion based on MPS using a modified version of the simulation algorithm developed by Zhang et al. (2006).

The necessity to infuse complex fine scale features in non-stationary coarse resolution images requires the development of new statistical downscaling methods. In this paper, we present a MPS simulation algorithm for downscaling coarse resolution images with trends. The approach is inspired by the concept of image pyramids introduced by Burt and Adelson (1983) for image compression. Here, the pyramid data structure is adapted for enhancing the spatial resolution of a given target coarse scale image. The missing fine scale structures are imported from a multi-resolution training image, which contains structural information at several scales. The spatial resolution of the target image is gradually enhanced through a series of conditional iterations of the downscaling algorithm. At each iteration, the algorithm generates features at a specific sub-pixel scale, such that the simulation of finer resolution features is conditioned to previously simulated coarser structures. This framework shares similarities with the multiple-grid approach proposed by Tran (1994). To address the presence of trends in the data set, at each scale, the input variable is decomposed into a trend and a residual component. The trend component is downscaled with a smooth deterministic interpolation technique. The residual component is downscaled using a quasi-pixelbased sequential simulation approach. Realizations of the sub-pixel residual variable are generated by integrating coarse and fine scale information with a probability aggregation operator. After the simulation of each pyramid level, the trend and residual components are summed back together, and the procedure is repeated at the next scale. We illustrate the methodology with the downscaling of digital elevation models (DEMs) in two mountain ranges in Switzerland. The algorithm is benchmarked against two-point and multiple-point statistics simulation techniques, as well as a deterministic interpolation method. Results are validated by a series of statistical and structural metrics.

The paper is organized as follows. In Section 2, we introduce the fundamental concepts of the methodology. The proposed downscaling algorithm is described in Section 3. In Section 4, we present the two case studies. The results are discussed in 
Section 5. Finally, in Section 6, we summarize the methodology and outline future work.

\section{Stochastic Downscaling}

This section presents the fundamentals of the stochastic downscaling method. Subsection 2.1 introduces the concept of representing multi-resolution imagery data as a stochastic spatial signal. This signal can be decomposed into a deterministic lowfrequency component (trend), and a stochastic higher-frequency component (residual). The term spatial frequency refers to a characteristic related to the scale of structural features on the image, which is interpreted as the inverse of structure scale. The trend component describes smooth large scale structures on an image, whereas the residual component represents small scale features. The downscaling of the trend and the residual components are presented in Subsection 2.2. Subsection 2.3 describes the conditional simulation of the fine scale residual variable with the sequential simulation formalism. Subsection 2.4 focuses on the estimation of local conditional probabilities from distances between conditioning and training data events. Finally, the probability aggregation method for integrating coarse and fine scale information is discussed in Subsection 2.5.

\subsection{Stochastic Representation of Imagery Data}

Let $z_{V}(\mathbf{u})$ be the sensor measurement of a physical property assigned to a coarse pixel $V$ centered at the location $\mathbf{u}$ on a target coarse resolution image denoted by $z_{V}$. In addition, let $z_{v}(\mathbf{u})$ be the small scale measurement of the same property on a fine pixel $v$ indexed on a target co-registered fine resolution image $z_{v}$. The coarse-to-fine resolution ratio between $z_{V}$ and $z_{v}$ is defined as $G=\sqrt{|V| /|v|}$, where $|V|$ and $|v|$ are the areas of the coarse and fine pixels, respectively. The notation is presented in Table 1.

In this paper, we make the assumption that any coarse datum $z_{V}\left(\mathbf{u}_{i}\right)$ corresponds to the linear average of the discrete set of $G^{2}$ co-registered fine resolution pixel values $\mathbf{z}_{v}\left(\mathbf{u}_{i}\right)=\left[z_{v}\left(\mathbf{u}_{g}\right), g=1, \ldots, G^{2}\right]$

$$
z_{V}\left(\mathbf{u}_{i}\right)=\frac{1}{G^{2}} \sum_{g=1}^{G^{2}} z_{v}\left(\mathbf{u}_{g}\right) \quad \forall i=1, \ldots, N
$$

where $\mathbf{z}_{v}\left(\mathbf{u}_{i}\right)$ is referred as a patch (a square array of fine scale pixel values) centered at the position $\mathbf{u}_{i}$ (Fig. 1), and $N$ is the total number of pixels on $z_{V}$. 
Table 1 Notation.

\begin{tabular}{ll}
\hline Notation & Description \\
\hline$\alpha$ & probability aggregation weight for fine scale data \\
$\beta$ & kernel weights normalizing constant \\
$\lambda\left(\mathbf{h}_{j}\right)$ & kernel weight as a function of $\mathbf{h}_{j}$ \\
$\sigma$ & kernel shape parameter \\
$\phi(\cdot)$ & distance-to-probability transformation function \\
$\Psi$ & dual-resolution training image \\
$\Omega$ & conditioning data \\
$\boldsymbol{\theta}$ & generic vector of algorithm parameters \\
$\boldsymbol{\Psi}$ & multi-resolution training image \\
$\mathbf{h}_{j}$ & coordinates offset of the $j$-th node from $\mathbf{u}$ \\
$\mathbf{D}(\mathbf{u})$ & local distance vector centered at $\mathbf{u}$ \\
$\mathbf{D}_{k}(\mathbf{u})$ & $k$-th element of the local distance vector \\
$\mathbf{R}_{v}(\mathbf{u})$ & multivariate fine residual RV centered at $\mathbf{u}$ \\
$\mathbf{r}_{v}(\mathbf{u})$ & fine residual patch centered at $\mathbf{u}$ \\
$\mathbf{u}$ & data location \\
$\mathbf{z}_{v}(\mathbf{u})$ & fine generic patch centered at $\mathbf{u}$ \\
$D(\cdot)$ & distance function \\
$d(\mathbf{u})$ & data event centered at $\mathbf{u}$ \\
$F(\cdot)$ & MPS simulation algorithm \\
$G$ & coarse-to-fine resolution ratio \\
$K$ & number of data events for computing the local CPDF \\
$m(\mathbf{u})$ & trend value centered at $\mathbf{u}$ \\
$m$ & trend image \\
$N$ & number of pixels on $z_{V}$ and $r_{V}$ \\
$n(\mathbf{u})$ & number of pixels in the search neighborhood centered at $\mathbf{u}$ \\
$q_{k}$ & rank of the $k$-th training data event \\
$R(\mathbf{u})$ & residual RV centered at $\mathbf{u}$ \\
$r(\mathbf{u})$ & residual value centered at $\mathbf{u}$ \\
$r$ & residual image \\
$(s)$ & superscript indicating simulated data \\
$(t)$ & superscript indicating training data \\
$V$ & subscript indicating coarse scale data \\
$v$ & subscript indicating fine scale data \\
$Z(\mathbf{u})$ & generic RV centered at $\mathbf{u}$ \\
$z(\mathbf{u})$ & generic datum value centered at $\mathbf{u}$ \\
$z$ & generic image \\
$\wedge$ & operator indicating estimated data \\
& \\
\hline & \\
\hline &
\end{tabular}

In geostatistics, $z_{V}(\mathbf{u})$ and $z_{v}(\mathbf{u})$ can be interpreted as realizations of the continuous RVs $Z_{V}(\mathbf{u})$ and $Z_{v}(\mathbf{u})$, respectively. The RV $Z(\mathbf{u})$ will be used to denote both $Z_{V}(\mathbf{u})$ and $Z_{v}(\mathbf{u})$ in expressions dealing with attributes at the same scale. Here, we propose to model $Z(\mathbf{u})$ as a spatial signal composed of two variables

$$
Z(\mathbf{u})=m(\mathbf{u})+R(\mathbf{u}),
$$

where $m(\mathbf{u})$ is a deterministic low-frequency signal (trend), and $R(\mathbf{u})$ is a RV representing its associated complementary stochastic higher-frequency signal (residual). 

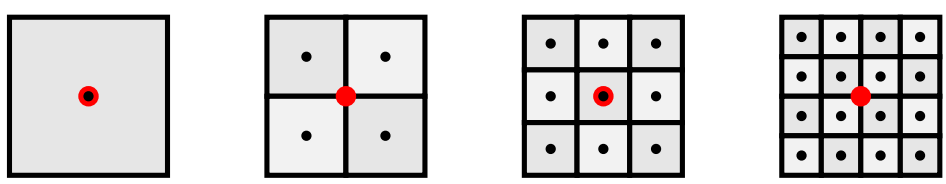

Fig. 1 Pixels' configuration for multiple coarse-to-fine resolution ratios. Left: reference coarse pixel $(G=$ 1). Middle left: co-registered patch of fine pixels $(G=2)$. Middle right: co-registered patch of fine pixels $(G=3)$. Right: co-registered patch of fine pixels $(G=4)$. Black dots indicate pixel centroids, red dots indicate patch centroids.

It is assumed that $R(\mathbf{u})$ is a spatially autocorrelated $R V$, that is, it has some sort of spatial structure.

An estimate of $m(\mathbf{u})$ might be obtained by applying an appropriate spatial lowpass filter on $z(\mathbf{u})$. The estimator $\hat{m}(\mathbf{u})$ is formulated as a weighted linear combination of $z(\mathbf{u})$ and its neighboring values $\left\{z\left(\mathbf{u}+\mathbf{h}_{j}\right), j=1, \ldots, n\right\}$ within a moving search window

$$
\hat{m}(\mathbf{u})=\sum_{j=0}^{n} \lambda\left(\mathbf{h}_{j}\right) z\left(\mathbf{u}+\mathbf{h}_{j}\right) \quad \text { with } \quad \sum_{j=0}^{n} \lambda\left(\mathbf{h}_{j}\right)=1,
$$

where $\mathbf{h}_{j}$ is the set of $n+1$ coordinates lag vectors radiating from $\mathbf{u}$, with $z(\mathbf{u})=$ $z\left(\mathbf{u}+\mathbf{h}_{0}\right)$ and $n \ll N$. The weights $\lambda\left(\mathbf{h}_{j}\right)$ are precomputed based on a kernel and set as function of $\mathbf{h}_{j}$. The value of $r(\mathbf{u})$, which is interpreted as a realization of $R(\mathbf{u})$, is the complement of $\hat{m}(\mathbf{u})$.

\subsection{Stochastic Downscaling of Images with Trends}

The goal of downscaling is to predict $z_{v}$ such that the prediction is coherent with $z_{V}$ and a given prior fine scale structural model. In order to access the uncertainty of such prediction, a stochastic approach for downscaling is proposed. The objective is to estimate the unknown true value $z_{v}(\mathbf{u})$ by generating $S$ realizations of $Z_{v}(\mathbf{u})$, denoted as $\left\{z_{v}^{(s)}(\mathbf{u}), s=1, \ldots, S\right\}$, conditioned to coarse resolution observations on $z_{V}$.

In practice, the fine scale variables $\hat{m}_{v}(\mathbf{u})$ and $r_{v}(\mathbf{u})$ cannot be directly computed because one has no knowledge of $z_{v}(\mathbf{u})$. As a result, $m_{v}(\mathbf{u})$ has to be estimated from neighboring coarse scale data. The sub-pixel trend estimator $\hat{m}_{v}(\mathbf{u})$ is thus obtained by downscaling $\hat{m}_{V}$ with a given deterministic interpolation method. Conversely, $R_{V}(\mathbf{u})$ is downscaled through stochastic simulation. A MPS simulation algorithm $F(\cdot)$ is used to generate conditional simulated realizations of $R_{v}(\mathbf{u})$, denoted as $r_{v}^{(s)}(\mathbf{u})$. This algorithm is parametrized by: a vector of parameters $\boldsymbol{\theta}$ asso- 
ciated with $F(\cdot)$, and a dual-resolution training image $\Psi$ which describes the spatial relationship between the coarse and fine scales

$$
R_{v}(\mathbf{u} \mid \Omega)=F(\boldsymbol{\theta}, \Psi \mid \Omega) \mapsto\left\{r_{v}^{(s)}(\mathbf{u} \mid \Omega), s=1, \ldots, S\right\}
$$

where $\mid \Omega$ refers to the conditioning to both coarse measurements assigned on $z_{V}$ and previously simulated fine scale data on $z_{v}$.

The dual-resolution training image is constructed from a pair of co-registered coarse and fine resolution images denoted by $z_{V}^{(t)}$ and $z_{v}^{(t)}$, respectively. It consists of an extensive multi-dimensional associative array listing all co-registered pairs of coarse and fine residual data events present on $z_{V}^{(t)}$ and $z_{v}^{(t)}$. The residual training variables, indicated by $r_{V}^{(t)}(\mathbf{u})$ and $r_{v}^{(t)}(\mathbf{u})$, respectively, are filtered out from $z_{V}^{(t)}(\mathbf{u})$ and $z_{v}^{(t)}(\mathbf{u})$ with Equation (2).

The simulated sub-pixel variable $z_{v}^{(s)}(\mathbf{u})$ is reconstructed by rewriting Equation (2) as follows

$$
z_{v}^{(s)}(\mathbf{u})=\hat{m}_{v}(\mathbf{u})+r_{v}^{(s)}(\mathbf{u})
$$

Figure 2 summarizes the methodology. Rounded white rectangles indicate the coarse resolution target image and the training data. Processes are represented as gray rectangles and intermediate data structures are depicted as rounded gray rectangles. The downscaled image corresponds to the rounded black rectangle. The preprocessing step, indicated by the dashed rounded rectangle, encompasses all the processes and data required for the construction of the dual-resolution training image. This step is performed only once. The spatial low-pass filtering and deterministic interpolation processes are identical for both target and training images.

\subsection{Downscaling with Sequential Simulation}

Let $\mathbf{R}_{v}\left(\mathbf{u}_{i}\right)=\left[R_{v}\left(\mathbf{u}_{g}\right), g=1, \ldots, G^{2}\right]$ denote the fine resolution multivariate continuous RV co-registered with $r_{V}\left(\mathbf{u}_{i}\right)$. Hence, the fine scale residual patch $\mathbf{r}_{v}\left(\mathbf{u}_{i}\right)$ is regarded as a joint realization of $\mathbf{R}_{v}\left(\mathbf{u}_{i}\right)$. The downscaling of $r_{V}\left(\mathbf{u}_{i}\right)$ is performed by generating a series of realizations of $\mathbf{R}_{v}\left(\mathbf{u}_{i}\right)$, denoted by $\mathbf{r}_{v}^{(s)}\left(\mathbf{u}_{i}\right)$, using sequential simulation (Goovaerts 1997). The multivariate conditional probability $\operatorname{Pr}\left\{\mathbf{R}_{v}\left(\mathbf{u}_{i}\right)=\right.$ $\left.\mathbf{r}_{v} \mid \Omega_{i-1}\right\}$ for $i=1, \ldots, N$ is given by the recursive Bayes relation 


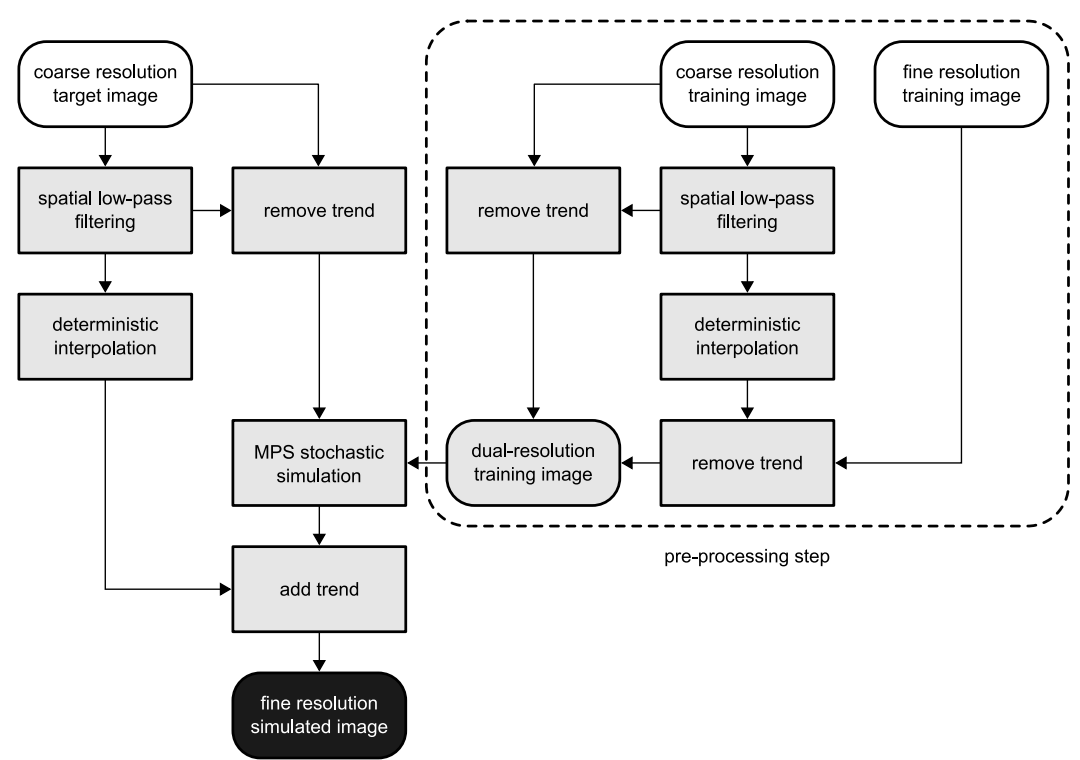

Fig. 2 Methodology flowchart. The rounded white rectangles indicate the input images. Processes are represented as gray rectangles and intermediate data structures are depicted as rounded gray rectangles. The output downscaled image corresponds to the rounded black rectangle.

$$
\begin{aligned}
& \operatorname{Pr}\left\{\mathbf{R}_{v}\left(\mathbf{u}_{1}\right)=\mathbf{r}_{v}, \ldots, \mathbf{R}_{v}\left(\mathbf{u}_{N}\right)=\mathbf{r}_{v} \mid \Omega_{N}\right\} \\
& =\operatorname{Pr}\left\{\mathbf{R}_{v}\left(\mathbf{u}_{1}\right)=\mathbf{r}_{v} \mid \Omega_{0}\right\} \cdot \prod_{i=2}^{N-1} \operatorname{Pr}\left\{\mathbf{R}_{v}\left(\mathbf{u}_{i}\right)=\mathbf{r}_{v} \mid \Omega_{i-1}\right\},
\end{aligned}
$$

where $\mid \Omega_{0}$ refers to the conditioning of the first iteration of the downscaling to the initial set of coarse observations, and $\mid \Omega_{i-1}$ to the conditioning of the $i$-th iteration to the $i-1$ previously simulated patches of fine pixels and the initial low-resolution data. The index sequence $i=1, \ldots, N$ defines the simulation path. The conditional probability $\operatorname{Pr}\left\{\mathbf{R}_{v}\left(\mathbf{u}_{i}\right)=\mathbf{r}_{v} \mid \Omega_{i-1}\right\}$ is approximated by the simulation algorithm $F(\cdot)$ based on $\Psi$

\subsection{Computing Local Conditional Probabilities}

Let $d_{V}\left(\mathbf{u}_{i}\right)$ denote the coarse resolution target data event centered at the location $\mathbf{u}_{i}$. This data structure is comprised of the central value $r_{V}\left(\mathbf{u}_{i}\right)$ and its $n_{V}$ neighboring coarse values

$$
d_{V}\left(\mathbf{u}_{i}\right)=\left\{r_{V}\left(\mathbf{u}_{i}+\mathbf{h}_{j}\right), j=0, \ldots, n_{V}\right\} .
$$


A larger set of coordinates lag vectors is used to retrieve the co-registered fine scale conditioning data event $d_{v}\left(\mathbf{u}_{i}\right)$

$$
d_{v}\left(\mathbf{u}_{i}\right)=\left\{r_{v}^{(s)}\left(\mathbf{u}_{i}+\mathbf{h}_{j}\right), j=1, \ldots, n_{v}\left(\mathbf{u}_{i}\right)\right\}
$$

where $r_{v}^{(s)}\left(\mathbf{u}_{i}+\mathbf{h}_{j}\right)$ corresponds to the current set of previously simulated fine pixels that are collocated with $d_{V}\left(\mathbf{u}_{i}\right)$ (Fig. 3).
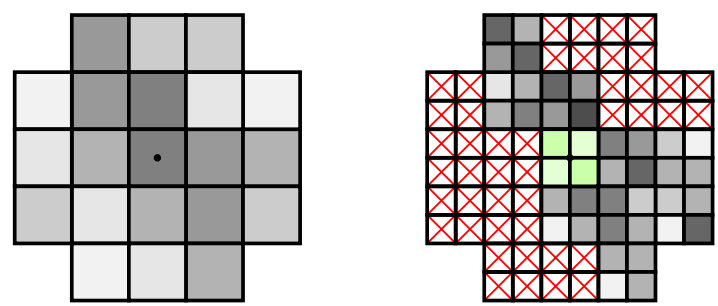

Fig. 3 Pair of co-registered coarse and fine scale conditioning data events $(G=2)$. Left: coarse scale data event. Right: incomplete fine scale data event. Black dots indicate the data events' centroids. White pixels with red crosses denote non-informed values, green pixels indicate locations to be simulated.

Conditioning to the coarse information in $d_{V}\left(\mathbf{u}_{i}\right)$ is achieved by restricting the sampling of training data events $d_{V}^{(t)}(\mathbf{u})$ that minimize the coarse scale distance function

$$
D\left(d_{V}\left(\mathbf{u}_{i}\right), d_{V}^{(t)}(\mathbf{u})\right)=\sum_{j=0}^{n_{V}} \lambda\left(\mathbf{h}_{j}\right)\left\|r_{V}\left(\mathbf{u}_{i}+\mathbf{h}_{j}\right)-r_{V}^{(t)}\left(\mathbf{u}+\mathbf{h}_{j}\right)\right\|_{2},
$$

where $\lambda\left(\mathbf{h}_{j}\right)$ are the weights from a given kernel. Note that a kernel function that provides higher values for $\lambda\left(\mathbf{h}_{0}\right)$ ensures better conditioning of the downscaling to the local measurement $z_{V}\left(\mathbf{u}_{i}\right)$.

The reproduction of fine scale spatial features is imposed by the minimization of the additional distance function

$$
D\left(d_{v}\left(\mathbf{u}_{i}\right), d_{v}^{(t)}(\mathbf{u})\right)=\sum_{j=1}^{n_{v}\left(\mathbf{u}_{i}\right)} \lambda\left(\mathbf{h}_{j}\right)\left\|r_{v}^{(s)}\left(\mathbf{u}_{i}+\mathbf{h}_{j}\right)-r_{v}^{(t)}\left(\mathbf{u}+\mathbf{h}_{j}\right)\right\|_{2}
$$

where $d_{v}^{(t)}(\mathbf{u})$ corresponds to a fine resolution training data event. Equation (10) ensures the sampling of compatible training data events by taking into account previously simulated fine scale data. 
In order to combine the two different sources of information given by Equations (9) and (10), both distances are converted into conditional probabilities. Most distance-based MPS simulation methods implicitly adopt a distance-to-probability transformation function. Simulation algorithms that rely on a threshold distance value as a criterion for accepting a given training data event, for example, assume a uniform local CPDF. As proposed by Hoffimann et al. (2017), the local CPDFs can also be defined as a function of the distances between data events. In this case, the transformation function $\phi(\cdot)$ needs to be defined explicitly such that conditional probabilities can be assigned to each candidate training data event. Probabilities are made inversely proportional to the distances to a given target data event.

The transformation function $\phi(\cdot)$ also has to take into account the relative dispersion of distance values within the local pool of candidates. If all the $K$ candidates are equally compatible with the conditioning data, the local CPDF should resemble a uniform distribution. In contrast, if only a small number of training data events is similar, the assignment of higher probabilities should be preferentially limited to this set of data events. This also applies to the opposite scenario (i.e. when several training data events are significantly dissimilar to the local conditioning data).

The coarse scale conditional probability is thus expressed as

$$
\operatorname{Pr}\left\{\mathbf{R}_{v}\left(\mathbf{u}_{i}\right)=\mathbf{r}_{v}^{(t)}\left(\mathbf{u}_{k}\right) \mid d_{V}\left(\mathbf{u}_{i}\right)\right\}=\phi\left(\mathbf{D}\left(\mathbf{u}_{i}\right)\right),
$$

where $\mathbf{r}_{v}^{(t)}\left(\mathbf{u}_{k}\right)$ refers to the central patch of $d_{v}^{(t)}\left(\mathbf{u}_{k}\right)$, and $\mathbf{D}\left(\mathbf{u}_{i}\right)$ is a $(K \times 1)$ local vector that stores the distances between $d_{V}\left(\mathbf{u}_{i}\right)$ and the set of $K$ best training data events $\left\{d_{V}^{(t)}\left(\mathbf{u}_{k}\right), k=1, \ldots, K\right\}$ (i.e. the training data events that minimize Eq. (9)). Note that Equation (11) is also used to estimate $\operatorname{Pr}\left\{\mathbf{R}_{v}\left(\mathbf{u}_{i}\right)=\mathbf{r}_{v}^{(t)}\left(\mathbf{u}_{k}\right) \mid d_{v}\left(\mathbf{u}_{i}\right)\right\}$.

\subsection{Integrating Coarse and Fine Scale Information}

To simulate structures from the training image that are compatible with the conditioning data, we integrate the local conditional probabilities derived from coarse and fine scale information with the log-linear pooling operator (Allard et al. 2012). The conditional probability $\operatorname{Pr}\left\{\mathbf{R}_{v}\left(\mathbf{u}_{i}\right)=\mathbf{r}_{v} \mid \Omega_{i-1}\right\}$ in Equation (6) is approximated by

$$
\begin{aligned}
& \operatorname{Pr}\left\{\mathbf{R}_{v}\left(\mathbf{u}_{i}\right)=\mathbf{r}_{v} \mid \Omega_{i-1}\right\} \approx \operatorname{Pr}\left\{\mathbf{R}_{v}\left(\mathbf{u}_{i}\right)=\mathbf{r}_{v}^{(t)}(\mathbf{u}) \mid d_{V}\left(\mathbf{u}_{i}\right), d_{v}\left(\mathbf{u}_{i}\right)\right\} \\
& =\operatorname{Pr}\left\{\mathbf{R}_{v}\left(\mathbf{u}_{i}\right)=\mathbf{r}_{v}^{(t)}\left(\mathbf{u}_{k}\right) \mid d_{V}\left(\mathbf{u}_{i}\right)\right\}^{1-\alpha_{i}} \cdot \operatorname{Pr}\left\{\mathbf{R}_{v}\left(\mathbf{u}_{i}\right)=\mathbf{r}_{v}^{(t)}\left(\mathbf{u}_{k}\right) \mid d_{v}\left(\mathbf{u}_{i}\right)\right\}^{\alpha_{i}}
\end{aligned}
$$

where $\alpha_{i}=\sum_{j=1}^{n_{v}\left(\mathbf{u}_{i}\right)} \lambda\left(\mathbf{h}_{j}\right)$. 
The weight $\alpha_{i}$ controls the relative importance of previously simulated fine resolution data during the aggregation process, based on the number of informed fine pixels and the kernel weights $\lambda\left(\mathbf{h}_{j}\right)$. The value of $\alpha_{i}$ changes throughout the simulation process. In the beginning of the simulation, conditional probabilities derived from coarse resolution data tend to dominate the shape of the aggregated CPDFs, however as the simulation progresses and $r_{v}^{(s)}$ becomes more populated, the importance of fine scale conditional probabilities gradually increases.

\section{Algorithm}

The following section aims at presenting the MPS simulation algorithm developed for downscaling. The simulation framework is later generalized as a multi-scale iterative process which allows the downscaling to handle large coarse-to-fine resolution ratios.

Algorithm 1 summarizes the downscaling of the target coarse resolution image $z_{V}$ using sequential simulation. The vector of algorithmic parameters $\boldsymbol{\theta}$ includes information related to the kernel function parameters for the spatial low-pass filters and distance functions, and the number of candidate training data events $K$ used for computing the local CPDFs. For reproducibility, a seed is used to initialize a pseudorandom number generator which defines the order of the simulation path and the sampling of local CPDFs. Multiple conditional simulated realizations are generated by feeding the algorithm different random seeds.

Initially, the residual image $r_{V}$ is extracted from $z_{V}$ with Equation (2), and the fine scale trend image $\hat{m}_{v}$ is estimated from $\hat{m}_{V}$ using a deterministic interpolation method. For each coarse pixel $r_{V}\left(\mathbf{u}_{i}\right)$ visited along the simulation path, the algorithm retrieves its corresponding pair of co-registered conditioning data events and computes the distance function in Equation (9) for all training data events stored in $\Psi$ with fast Fourier transform (FFT) (Kwatra et al. 2003). The $K$ best training data events are then sorted in ascending order according to the coarse scale distance function. Subsequently, the distances between $d_{V}\left(\mathbf{u}_{i}\right)$ and this subset of training data events are stored in $\mathbf{D}\left(\mathbf{u}_{i}\right)$, which is later used to estimate the local CPDF. The same procedure is repeated for the co-registered fine resolution conditioning data event, however, only for those $K$ preselected locations. Once the simulation is finished, $z_{v}^{(s)}$ is restored with Equation (5) (line 12 of Algorithm 1).

When the coarse-to-fine resolution ratio is large (e.g. $G>3$ ), Algorithm 1 has to be adapted to allow a better reproduction of the different structures that can be found over a range of scales in $r_{v}^{(t)}$. To this end, we adopt a multi-scale iterative process based on smaller magnification factors. The downscaling of $z_{V}$ is done through a series of hierarchical conditional simulations. This is accomplished by constraining simulations at finer resolutions to previously simulated coarser outputs. The process is interrupted when the downscaled image reaches a target spatial resolution of size $\left|v^{\prime}\right|$ which is the fine pixel size of the finest dual-resolution training image available. 


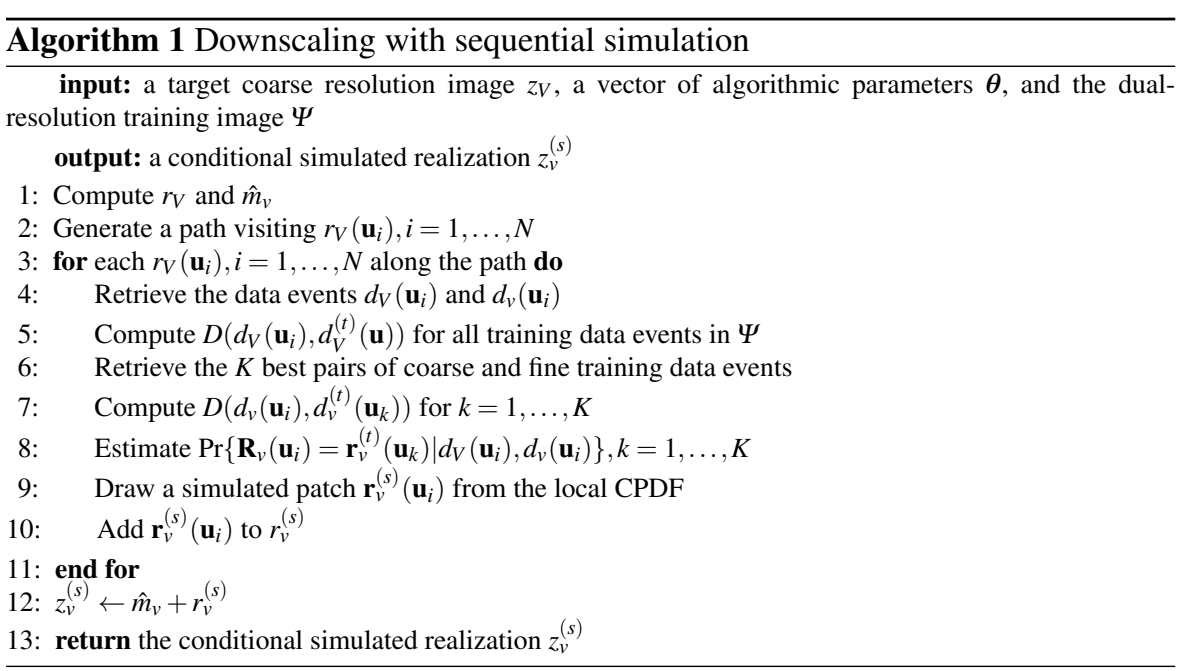

Note that this iterative procedure entails replacing $\Psi$ with a vector of dual-resolution training images.

The multi-scale downscaling of the target image $z_{V}$ is summarized in Algorithm 2. The simulation of the sub-pixel residual variable is performed at multiple scales based on a series of conditional iterations of Algorithm 1. At the end of each iteration, the output realization $z_{v}^{(s)}$ is assigned as the new target coarse scale image (line 3 of Algorithm 2). The process is repeated until the desired target spatial resolution is reached.

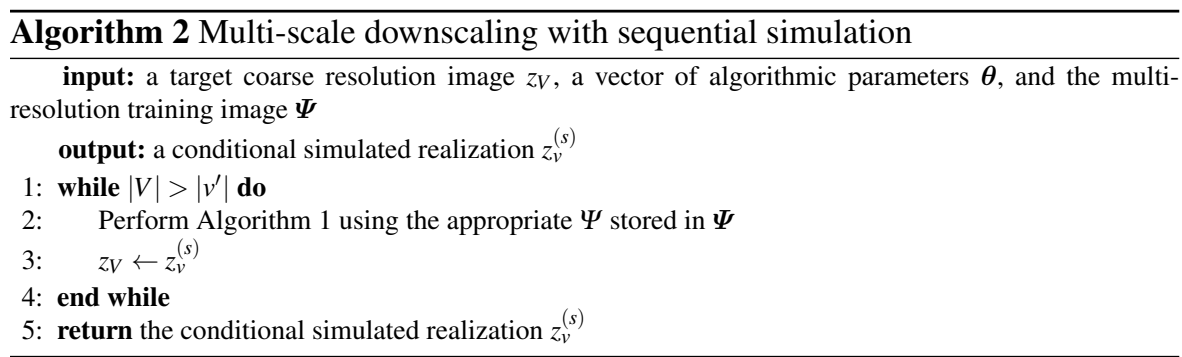

\section{Case Studies}

The methodology is demonstrated with illustrative examples using DEMs from two mountain ranges in Switzerland. Our MPS-based downscaling method is used to generate fine resolution conditional simulations. The coarse and fine resolution DEMs of 
both study areas consist of coarsened versions of the Swisstopo swissALTI3D DEM (Wiederkehr and Möri 2013) produced with linear upscaling. Although there is a natural degree of similarity between both data sets as they originate from universal tectonic and surface processes, such as orogeny and mass wasting, they represent very distinct geomorphological settings. The maximum amplitude of the trend component in the two case studies is vastly different: In the Western Alps example it reaches 1.5 $\mathrm{km}$, whereas in the Jura Mountains case it is only $300 \mathrm{~m}$. The two mountain ranges are also characterized by contrasting landforms. The Western Alps are dominated by steep gradients, debris cones, and braided drainage systems, while the Jura Mountains are predominately karsts with lower gradients mainly driven by dissolution processes.

In Subsection 4.1, we define the kernel used for generating the weights for the spatial low-pass filter and distance functions as well as the distance-to-probability transformation function. The estimation of the sub-pixel trend image and the construction of multi-resolution training images are also discussed. Subsection 4.2 describes the setup of the other downscaling methods used for benchmarking. The statistical and structural metrics used to validate the results are discussed in Subsection 4.3. Subsection 4.4 presents an example using DEMs of the Western Alps, and Subsection 4.5 illustrates the method with DEMs from the Jura Mountains.

\subsection{Kernels and Distance-to-Probability Transformation Function}

In both case studies, a normalized Gaussian radial basis function is used for computing the kernel weights for the spatial low-pass filter (Eq. (3)) and distance functions (Eqs. (9) and (10))

$$
\lambda\left(\mathbf{h}_{j}\right)=\frac{1}{2 \pi \sigma^{2} \beta} \exp \left(-\frac{\left\|\mathbf{h}_{0}-\mathbf{h}_{j}\right\|_{2}}{2 \sigma^{2}}\right)
$$

where $\sigma$ is the kernel shape parameter, and $\beta$ is the normalizing constant (i.e. the sum of all kernel weights).

The transformation function $\phi(\cdot)$ assigns conditional probabilities to the local pool of candidate training data events. This function should be flexible enough to allow the sampling of either a large or a small portion of the $K$ candidate training data events. The availability of a large pool of candidates for sampling is desirable for generating sub-pixel variability in the simulated realizations. However, a more thorough sampling might be important to enforce the reproduction of less frequent features present in the training image.

Based upon the aforementioned criteria, we formulated the following distanceto-probability transformation function 


$$
\phi\left(\mathbf{D}_{k}\left(\mathbf{u}_{i}\right)\right)=\left(\frac{\mathbf{D}_{k}\left(\mathbf{u}_{i}\right)-\min \left(\mathbf{D}\left(\mathbf{u}_{i}\right)\right)}{\max \left(\min \left(\mathbf{D}\left(\mathbf{u}_{i}\right)\right), c\right)}+1\right)^{-q_{k}}
$$

where $\mathbf{D}_{k}\left(\mathbf{u}_{i}\right)=D\left(d_{V}\left(\mathbf{u}_{i}\right), d_{V}^{(t)}\left(\mathbf{u}_{k}\right)\right)$ or $D\left(d_{v}\left(\mathbf{u}_{i}\right), d_{v}^{(t)}\left(\mathbf{u}_{k}\right)\right), c$ is a small constant inserted in the denominator to avoid division by zero, and $q_{k}$ corresponds to the rank of $d_{V}^{(t)}\left(\mathbf{u}_{k}\right)$ after the sorting operation.

Note that Equation (14) allows one to consider a variable number of candidate training data events at each location to be simulated. If all the distances stored in $\mathbf{D}\left(\mathbf{u}_{i}\right)$ are similar, a larger set of the $K$ training data events is considered for sampling. Conversely, if such distances are significantly dissimilar, only the most compatible data events are likely to be drawn. The numerator of the base term in Equation (14) measures the dispersion within the pool of candidates by computing the difference in distance units between all the $K$ elements against the best candidate training data event. The denominator converts the absolute values into relative measurements towards the smallest element in the set. The exponent $-q_{k}$ scales the base such that higher conditional probabilities are assigned to the training data events that minimize the numerator. Adding +1 to the base term allows assigning uniform probabilities if all candidate training data events have roughly the same distance to the target data event. Computed conditional probabilities are later re-scaled to sum up to one.

The algorithm is driven by three parameters: one kernel shape parameter for the spatial low-pass filter denoted by $\sigma_{F}$, another shape parameter for the distance functions $\sigma_{D}$, and the number of candidate training data events $K$. Additionally, we compute $\hat{m}_{v}(\mathbf{u})$ and $\hat{m}_{v}^{(t)}(\mathbf{u})$ by downscaling the coarse scale trend estimates with bicubic interpolation. In both examples, the multi-resolution training images are built directly from $z_{v}^{(t)}$ by linear upscaling. At each scale, the decomposition between trend and residual is done using a spatial low-spatial filter with a radius that is proportional to the pixel size of the current coarse scale. The sequential simulation process is performed using a random path.

\subsection{Benchmarking Against Other Techniques}

The proposed algorithm is benchmarked against the two-point statistics area-to-point simulation method (Kyriakidis and Yoo 2005), the direct sampling MPS simulation algorithm (Mariethoz et al. 2010), and the bicubic interpolation method (Keys 1981). In order to carry a fair comparison between techniques, $z_{V}$ is detrended prior to simulation. Realizations are conditioned to both $r_{V}$ and previously simulated fine resolution data. The downscaled DEMs are then restored by addition of the estimated trend component $\hat{m}_{v}$ computed with bicubic interpolation. 
The downscaling by area-to-point simulation is performed with the error simu362 lation framework (Journel and Huijbregts 1978 Liu and Journel 2009). As the simu3 lation paradigm only applies to Gaussian variables, the reproduction of the fine scale target histogram must be achieved through post-processing. The empirical CDF of $r_{v}^{(t)}$ is used as source distribution for a normal score transform. The histogram transformation morphs this empirical CDF into a zero mean Gaussian distribution with unit variance through quantile mapping. An artificial coarse scale Gaussian variable is constructed through linear upscaling of the transformed version of $r_{v}^{(t)}$. The histogram transformation is then applied to $r_{V}$ using the previous Gaussian distribution as target CDF. Note that this approximation inherently introduces conditioning errors since the upscaling function between the original coarse and fine resolution residuals is actually non-linear. Unlike the trended component, each coarse residual pixel value does not necessarily corresponds to the arithmetic mean of its co-registered fine residual patch due to the trend removal operation. Unconditional fine resolution realizations of a zero mean Gaussian process are generated with the FFT moving average simulation algorithm (Ravalec et al. 2000). The inference of the fine scale (i.e. pointsupport) variogram model is carried out as a two-step process. The first part consists of inferring the shape of the variogram model near the origin (i.e. for lags smaller than the coarse pixel size). This is performed based on the Gaussian transform of $r_{v}^{(t)}$. The second step is the inference of the variogram model geometric anisotropy, which is calibrated based on the transformed version of $r_{V}$. Each conditional realization is then back-transformed into the original variable space using the empirical CDF of $r_{v}^{(t)}$ as target distribution.

Downscaling with the direct sampling algorithm can be seen as a conditional simulation problem with an exhaustive secondary variable. The two required preprocessing steps are the resampling of the coarse scale DEMs (in order to have colocated neighbors for both primary and secondary variables) and the variable normalization operations. In this study, $r_{V}$ and $r_{V}^{(t)}$ are resampled at the fine scale pixel size using nearest neighbor interpolation. The target and training residual DEMs are normalized using a min-max scaling. The minimum and maximum values are extracted from the training data. After simulation, output realizations are re-scaled.

Although not a geostatistical technique, the bicubic interpolation method is widely used in practical applications owing to its capability for generating smooth surfaces with a short processing time. Interpolations are performed based solely on $z_{V}$. Its application to the data sets hereby studied is straightforward, and it provides a reference point for comparison and analysis of the results.

\subsection{Validation}

The downscaled DEMs are evaluated based on a series of statistical and structural metrics. The reproduction of the reference fine scale terrain elevation probability dis- 
tribution is verified with empirical cumulative distribution functions (CDFs). The conditioning quality of the simulations to the input coarse data is quantified based on the average mean error (ME) and root-mean-square error (RMSE) between the reference coarse resolution DEMs and the upscaled realizations. The structural accuracy of the downscaling is assessed by computing empirical variograms, high-order cumulant maps (Dimitrakopoulos et al. 2010), probability of connection curves, and the mean structural similarity (SSIM) index (Wang et al. 2004) between simulated realizations and the reference residual DEMs. The topology of the realizations is described using the Euler characteristic. Detailed information about the probability of connection function and the Euler characteristic, and their application for the evaluation of continuous random fields can be found in Renard and Allard (2013). All the validation metrics, with exception of the ME and RMSE, are computed on the residual DEMs to remove the effect of large scale topographic structures.

\subsection{The Western Alps Example}

This example considers DEMs from a portion of the Western Swiss Alps. The topography in this region is characterized by a rough terrain with steep natural slopes, high altitude peaks, and glacially carved valleys. The reference DEMs and their respective residual DEMs are shown in Figure 4. The coarse DEM has dimensions of $64 \times 64$ pixels, and each pixel has size of $32 \times 32 \mathrm{~m}$, which is approximately the spatial resolution of the 1-arc second near-global DEM produced from NASA's Shuttle Radar Topography Mission (SRTM). The medium and fine DEMs have dimensions of $128 \times 128$ pixels and $256 \times 256$ pixels, with pixel sizes of $16 \times 16 \mathrm{~m}$ and $8 \times 8 \mathrm{~m}$, respectively. The footprint of the DEMs is roughly $4 \mathrm{~km}^{2}$. The coarse DEM is used for conditioning, while the medium and fine resolution DEMs are used for validation of the simulations. The residual DEMs were computed using a spatial low-pass filter with $\sigma_{F}=64 \mathrm{~m}$. Negative relief features in the residual variable represent gullies and drainage networks, whereas positive relief structures correspond to cliffs and mountain ridges.

Figure 5 illustrates the training DEMs and their respective residual DEMs. These DEMs are from a neighboring area that shares similar topographic features with the reference data set. The training data set has a significantly larger footprint than the target area $\left(\approx 16 \mathrm{~km}^{2}\right)$. The training DEMs should be extensive enough to include the expected range of relevant structural patterns to be determined. The coarse, medium, and fine resolution DEMs have the following dimensions: $128 \times 128$ pixels, $256 \times 256$ pixels, $512 \times 512$ pixels, respectively, with the same pixel size configuration of the reference data set. The training residual DEMs are displayed using the same spatial low-pass filter setup. Table 2 lists summary statistics from the target and training coarse resolution DEMs used in both case studies. Note that all residual DEMs show similar mean values, but the training DEMs have higher variance and range than their corresponding target data sets. 

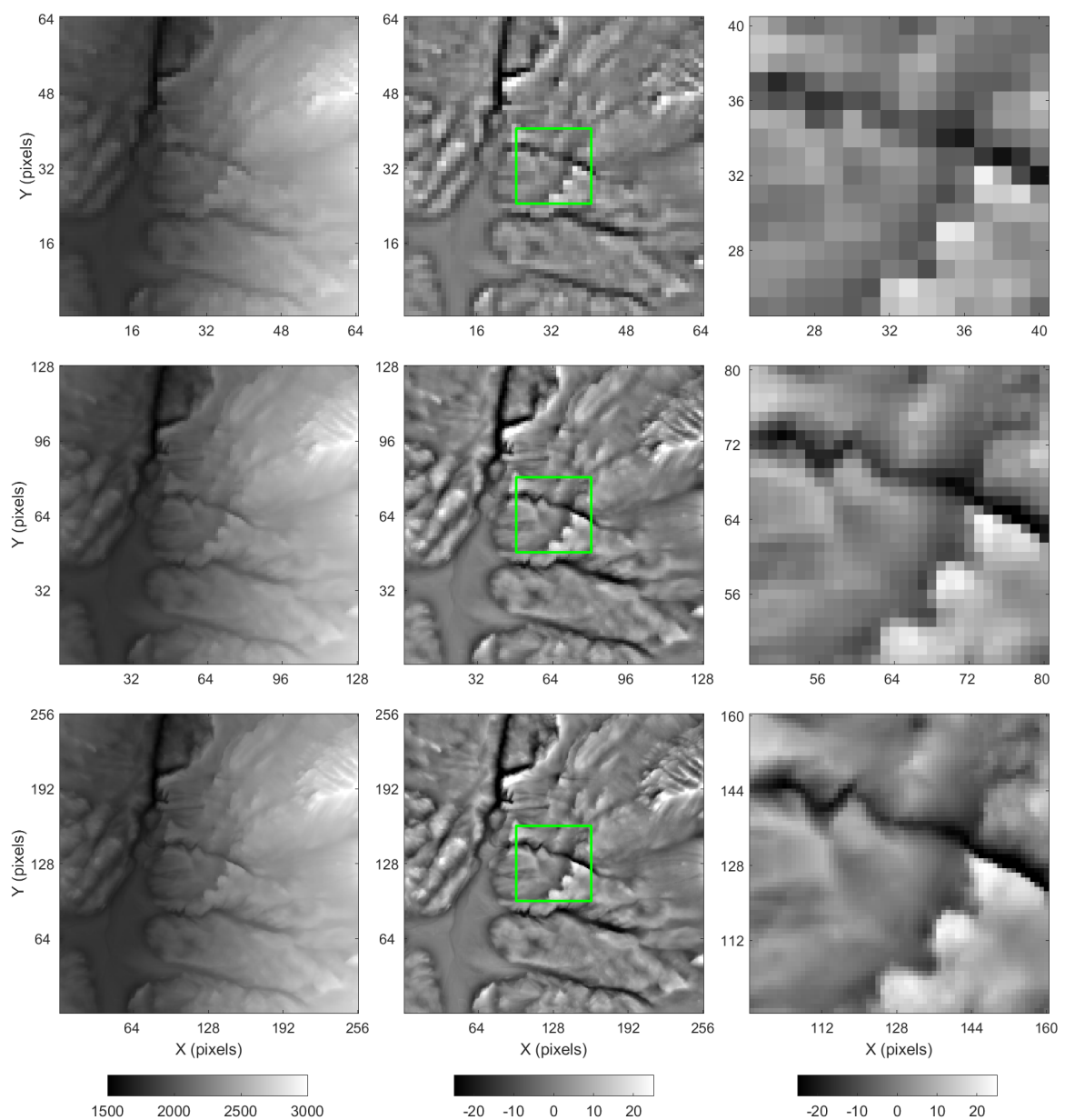

Fig. 4 The Western Alps reference DEMs. Top left: coarse resolution DEM. Top center: coarse resolution residual DEM. Top right: coarse resolution zoom. Middle left: medium resolution DEM. Middle center: medium resolution residual DEM. Middle right: medium resolution zoom. Bottom left: fine resolution DEM. Bottom center: fine resolution residual DEM. Bottom right: fine resolution zoom. The residual component of the trended DEMs has a vertical exaggeration factor of 8x. Green boxes highlight the magnified area. Colorbars' unit is meter.

An ensemble of 20 simulated realizations with a magnification factor of $4 \mathrm{x}$ was generated based on two iterations of the algorithm. Since the pixel sizes of the multiresolution training DEMs are all multiples of 2, the magnification factor per iteration was set equal to $G=2$. The search window used by the spatial low-pass filter and the retrieval of data events at the coarse scale has dimensions of $5 \times 5$ pixels. The size of the corresponding fine resolution search window is $10 \times 10$ pixels.

The parameters used for the two iterations of the downscaling of the Western Alps example are listed in Table 3. Parameters were chosen such that the algorithm 
Table 2 Summary statistics of the target and training coarse resolution DEMs from the Western Alps and the Jura Mountains examples. Values are in residual elevation (in meters).

\begin{tabular}{lrrrr}
\hline & mean & std. dev. & min. & max. \\
\hline Western Alps (target) & -0.38 & 5.94 & -34.71 & 31.19 \\
Western Alps (training) & -0.11 & 7.81 & -57.04 & 60.26 \\
\hline Jura Mountains (target) & -0.05 & 2.84 & -14.66 & 13.11 \\
Jura Mountains (training) & -0.03 & 4.30 & -18.00 & 27.66 \\
\hline
\end{tabular}

performs reasonably well for both data sets. Typically, they may be inferred from the dual-resolution training image through cross-validation. The spatial low-pass filter kernel shape parameter $\sigma_{F}$ is calibrated in order to generate an auto-correlated residual. The simulation of the fine resolution residual component is only feasible if the spatial low-pass filter is applied to $z_{V}$ prior to its interpolation. This leads to a significant improvement in the structural accuracy of the simulated realizations. The distance functions' kernel shape parameter $\sigma_{D}$ is set such that the central pixel of the coarse scale data events receives roughly half of the sum of the kernel weights. The weight given to the central pixel directly affects the conditioning quality of the downscaling to the target DEM. The number of candidate training data events $K$ is adjusted to achieve a trade-off between the structural accuracy of the simulated realizations, sub-pixel variability, and computational efficiency.

Table 3 Algorithm parameters used in the Western Alps and Jura Mountains examples.

\begin{tabular}{llrr}
\hline Parameter & Unit & $G=2$ & $G=4$ \\
\hline$\sigma_{F}$ & $\mathrm{~m}$ & 64 & 32 \\
$\sigma_{D}$ & $\mathrm{~m}$ & 16 & 8 \\
$K$ & - & 20 & 20
\end{tabular}

The downscaling by area-to-point simulation is performed using a global search neighborhood. The fitted variogram model consists of a normalized anisotropic $k$ Bessel model whose parameters are listed in Table 4. The direct sampling algorithm parameters are configured to enforce the scanning of the entire training image. The normalized acceptance threshold is set to a small value to maximize the structural quality of the output realizations. Table 5 lists the algorithm parameters used for the Western Alps and Jura Mountains examples. A standard configuration setup is employed which includes the use of an isotropic search radius for the retrieval of data events, and the $L^{2}$ norm for distance computation. The exponent in the distance function is set to zero. For a detailed description of the algorithm and its parameters, the reader is referred to Mariethoz et al. (2010) and Meerschman et al. (2013).

Figures 6-9 illustrate two realizations and interpolations of the sub-pixel DEMs and their corresponding residual topographies generated with the four benchmarked 
Table 4 Area-to-point simulation parameters used in the Western Alps and Jura Mountains examples.

\begin{tabular}{lllrr}
\hline Example & Parameter & Unit & $G=2$ & $G=4$ \\
\hline \multirow{4}{*}{ Western } & $\sigma_{F}$ & $\mathrm{~m}$ & 64 & 64 \\
Alps & Variogram model & - & $k$-Bessel & $k$-Bessel \\
& Max. range & $\mathrm{m}$ & 22.4 & 20.8 \\
& Min. range & $\mathrm{m}$ & 19.2 & 17.6 \\
& Azimuth & degrees & 90 & 90 \\
& Shape parameter & - & 2 & 2 \\
\hline \multirow{4}{*}{ Jura } & $\sigma_{F}$ & $\mathrm{~m}$ & 64 & 64 \\
Mountains & Variogram model & - & $k$-Bessel & $k$-Bessel \\
& Max. range & $\mathrm{m}$ & 65.6 & 65.6 \\
& Min. range & $\mathrm{m}$ & 40.0 & 40.0 \\
& Azimuth & degrees & 90 & 90 \\
& Shape parameter & - & 1 & 1 \\
\hline
\end{tabular}

Table 5 Direct sampling algorithm parameters used in the Western Alps and Jura Mountains examples.

\begin{tabular}{lllrr}
\hline Variable & Parameter & Unit & $G=2$ & $G=4$ \\
& $\sigma_{F}$ & $\mathrm{~m}$ & 64 & 64 \\
& Training image scanning fraction & - & 1.0 & 1.0 \\
\hline \multirow{3}{*}{ Coarse } & Normalized acceptance threshold & - & 0.005 & 0.005 \\
& Max. search radius & number of pixels & 5 & 10 \\
& Max. neighborhood size & number of pixels & 9 & 21 \\
\hline \multirow{3}{*}{ Fine } & Normalized acceptance threshold & - & 0.005 & 0.005 \\
& Max. search radius & number of pixels & 5 & 10 \\
& Max. neighborhood size & number of pixels & 8 & 20
\end{tabular}

techniques. Summary statistics for the downscaling results are listed in Table 6. Statistical and structural validation metrics for the realizations and interpolations are depicted in Figures 10-13 and Table 7. The empirical CDFs, directional variograms, Euler characteristic, and connectivity curves for the proposed method, area-to-point simulation, and the direct sampling algorithm are displayed as min-max envelopes generated from 20 realizations (Figs. 10 and 11). Statistics and validation metrics calculated from simulated realizations consist of the mean values plus or minus one standard deviation.

Statistically, the medium and fine resolution DEMs generated with the proposed downscaling algorithm are the closest to the reference data set when compared to the realizations produced by other techniques (Table 6). However, a systematic underestimation of the reference standard deviation values is observed. Figure 6 illustrates reproduction of low entropy patterns characterized by the spatial connectivity between high and low residual elevation values. These structures can be observed in the reference fine resolution residual DEM (Fig. 4). The area-to-point simulation realizations overestimate the standard deviation and spread of the reference residual DEMs (Table 6). This is likely a consequence of the mismatch between the probability dis- 
tributions of the fine scale residuals from the training image and the reference data set. The histogram transformations are based on the empirical CDF of the fine resolution training image which has a larger range than the fine scale reference histogram (Table 6). As expected, area-to-point simulation realizations have a higher degree of spatial entropy. Simulated textures tend to disconnect high and low residual elevation values (Fig. 7). Realizations also exhibit high-frequency structures in areas that are predominantly bland in the reference fine resolution residual DEM (Figs. 4 and 7). The direct sampling realizations have slightly lower variance than the results produced by the proposed algorithm. The bicubic interpolation predictions consist of blurred representations of the fine scale reference DEM (Fig. 9). The spatial smoothing caused by the interpolation process is also observed in the summary statistics, characterized by the underestimation of the variance and the sample minimum and maximum (Table 6).

On average, the proposed algorithm generates fine resolution terrain models that are consistent with the coarse scale conditioning data. The average ME and RMSE between the reference coarse resolution DEM and the upscaled realizations are smaller than the ones produced by the direct sampling and bicubic interpolation (Table 7). The area-to-point simulation realizations have the smallest RMSE for both magnification factors. However, they produce similar ME values. The scatter plots in Figs. 10 and 11 reveal an unbiased dispersion between the reference coarse resolution residual elevation and the coarse scale conditioning error for realizations generated with the proposed algorithm. The direct sampling error dispersion is somewhat higher. Negative correlation between the reference residual elevation and the conditioning errors for the upper and lower ends is observed (Figs. 10 and 11). In contrast, area-to-point simulations provide precise reproduction of intermediate coarse resolution measurements but they generate a positive correlation towards low and high values. The bicubic interpolation results clearly show a negative correlation between the conditioning errors and the coarse residual elevation (Figs. 9 and 10).

Structural validation metrics reveal that the proposed downscaling method is more effective at reproducing the reference fine scale variability and sub-pixel structures. This is reflected in the agreement between the simulations and the reference data in the directional variograms, Euler characteristic, and probability of connection plots (Figs. 10 and 11). Area-to-point simulations generate more variability and are unable to reproduce the spatial connectivity of fine scale residuals. It is also evident that the bicubic interpolation method underestimates the sub-pixel variability and does not reproduce the topology and the connectivity of the reference residual DEMs. The direct sampling realizations tend to generate less variability when compared to the proposed algorithm and have issues for reproducing the Euler characteristic curves for residual elevation values within the interval between -10 and $10 \mathrm{~m}$ for $G=4$. However, it is worth noting that the proposed algorithm seems to underestimate the reference negative Euler number values for residual elevation thresholds within the interval from -10 to $10 \mathrm{~m}$ (Figs. 9 and 10). In addition, similarly to the other stochastic techniques, simulated realizations tend to produce erratic fluctuations in the probability of connection for residual elevation values under $-15 \mathrm{~m}$ and over 
$15 \mathrm{~m}$ for $G=4$ (Fig. 10). This is most likely to be due to random noise inherent to the simulation process and its respective propagation across scales (i.e. in the following iterations). As a result, the structural accuracy of the realizations is expected to deteriorate at higher magnification factors.

The bicubic interpolation estimates have the highest mean SSIM for both the medium and fine resolution predictions whereas the area-to-point simulated realizations display the smallest scores (Table 7). Stochastic methods will generally yield lower SSIM because, by construction, they do not aim to minimize the local variance of the predictions. The bicubic interpolation produces smooth surfaces devoid from noise. Withal, the resulting textures are deprived from the sub-pixel patterns imported from the dual-resolution training image. Figures 12 and 13 show the mean absolute error (MAE) between the simulated and the reference sub-pixel residual elevation third and fourth-order cumulant maps. The spatial templates used for computing the experimental cumulants are displayed next to the maps. As expected, the MPS-based approaches outperform the other two methods. The MAE generated at short lags configurations by both MPS methods are approximately one order of magnitude smaller than the MAE produced by area-to-point simulation and bicubic interpolation. The proposed approach tends generate larger small scale errors in the third-order cumulant map compared to the direct sampling algorithm. This is likely caused by edge artifacts between adjacent simulated patches. Nevertheless, the scenario is reversed in the fourth-order cumulant MAE map.

Table 6 Summary statistics of the downscaled DEMs from the Western Alps example. Values are in residual elevation (in meters)

\begin{tabular}{lrrrrr}
\hline & $G$ & mean & std. dev. & min. & max. \\
\hline Training & 2 & -0.12 & 8.99 & -63.69 & 72.44 \\
Reference & 2 & -0.45 & 6.83 & -40.54 & 37.37 \\
Proposed method & 2 & $-0.44 \pm 0.00$ & $6.67 \pm 0.01$ & $-40.08 \pm 1.16$ & $37.35 \pm 1.69$ \\
Area-to-point & 2 & $-0.44 \pm 0.00$ & $7.10 \pm 0.01$ & $-54.63 \pm 2.89$ & $55.96 \pm 3.20$ \\
Direct sampling & 2 & $-0.45 \pm 0.00$ & $6.57 \pm 0.02$ & $-39.32 \pm 2.01$ & $38.74 \pm 2.92$ \\
Bicubic & 2 & -0.45 & 6.19 & -34.62 & 31.48 \\
\hline Training & 4 & -0.11 & 8.89 & -63.33 & 85.03 \\
Reference & 4 & -0.41 & 6.76 & -41.43 & 37.36 \\
Proposed method & 4 & $-0.41 \pm 0.00$ & $6.55 \pm 0.02$ & $-42.07 \pm 1.98$ & $40.07 \pm 2.38$ \\
Area-to-point & 4 & $-0.41 \pm 0.00$ & $7.06 \pm 0.02$ & $-57.93 \pm 2.84$ & $60.96 \pm 3.80$ \\
Direct sampling & 4 & $-0.42 \pm 0.00$ & $6.42 \pm 0.01$ & $-43.59 \pm 1.52$ & $40.39 \pm 1.90$ \\
Bicubic & 4 & -0.42 & 5.95 & -35.23 & 31.38
\end{tabular}

\subsection{The Jura Mountains Example}

The second case study uses DEMs from a subset of the Jura Mountains. This subalpine mountain range is characterized by karst topography and relative low-gradient 
Table 7 Validation of the Western Alps example.

\begin{tabular}{lrrrr}
\hline & $G$ & ME $(\mathrm{cm})$ & RMSE $(\mathrm{cm})$ & SSIM \\
\hline Proposed method & 2 & $0.90 \pm 0.76$ & $58.62 \pm 1.17$ & $0.914 \pm 0.001$ \\
Area-to-point & 2 & $-1.24 \pm 0.37$ & $40.63 \pm 0.94$ & $0.883 \pm 0.003$ \\
Direct sampling & 2 & $1.50 \pm 0.82$ & $86.43 \pm 1.50$ & $0.893 \pm 0.002$ \\
Bicubic & 2 & 1.33 & 68.05 & 0.932 \\
\hline Proposed method & 4 & $1.13 \pm 0.80$ & $60.15 \pm 1.00$ & $0.877 \pm 0.002$ \\
Area-to-point & 4 & $-1.44 \pm 0.58$ & $46.98 \pm 1.18$ & $0.832 \pm 0.002$ \\
Direct sampling & 4 & $1.24 \pm 0.66$ & $67.17 \pm 1.20$ & $0.875 \pm 0.001$ \\
Bicubic & 4 & 1.68 & 79.45 & 0.897 \\
\hline
\end{tabular}

landforms when compared to the Western Alps example. Figure 14 illustrates the reference DEMs and the residual terrain elevation models. The training trended and residual DEMs from a neighboring analog area are depicted in Figure 15. The spatial low-pass filter configuration for obtaining the residuals, the DEMs dimensions, pixel sizes, footprints are identical to the ones presented in Subsection 4.4.

A set of 20 conditional simulations was generated using the same multi-scale iterative downscaling approach and parameters setup used in the Western Alps case study (Table 3). The area-to-point simulation and the direct sampling algorithm parameters used for this example are listed in Tables 4 and 5, respectively. Simulated realizations and estimates are shown in Figures 16-19. Summary statistics are listed in Table 8. The statistical validation metrics are depicted in Table 9 and Figures 2023.

The results for the Jura Mountains example confirm the ones from the Western Alps case study. The proposed MPS algorithm outperforms the other techniques for the majority of validation metrics. The method is able to reproduce relatively well the fine scale terrain elevation probability distributions for both magnification factors (Table 8 and Figs. 20, 21). The area-to-point simulated realizations generate more variability than the reference data set. Similarly to the previous example, this is most likely caused by the reliance on the training image fine scale empirical CDF for the histogram transform. The conditioning ME, RMSE in Table 9 and the error dispersions displayed in the scatter plots for both medium (Fig. 20) and fine resolution (Fig. 21) predictions are akin to the results presented in Subsection 4.4, although the magnitude of the errors is significantly smaller. The proposed approach generates the smallest ME for both magnification factors, and the highest SSIM values among the stochastic simulation methods (Table 9).

The structural accuracy of the downscaled DEMs produced by the different techniques are also akin to the Western Alps case study. Notwithstanding, the loss of fine scale variability is significantly less pronounced in this example. The relative differences between the standard deviations of the reference and simulated DEMs is approximately halved (Table 8 ). This can also be observed in the improved match between the empirical variograms (Figs. 20 and 21). The Euler characteristic and 
probability of connection plots indicate that the proposed algorithm generates less random noise. Underestimation of negative Euler numbers within the range of -5 to $5 \mathrm{~m}$ is evident after two iterations of the algorithm. However, the erratic fluctuations in the connectivity curves for small and large residual elevations are much less pronounced (Fig. 21). The noise reduction might be related to the fact that the topography in this region is not as rugged as in the Western Alps. The spatial patterns in the training DEMs are generally smoother and, consequently, less noise is propagated throughout the simulation process. The area-to-point simulation and the bicubic interpolation method are unable to the reproduce the fine scale variability present in the reference data, and cannot adequately mimic the topology and the spatial connectivity of the sub-pixel residual variable (Figs. 20 and 21). The proposed algorithm and the direct sampling realizations tend to produce similar Euler characteristic and connectivity curves for $G=2$ (Figs. 20). However, these curves start to differentiate themselves when $G=4$. The proposed algorithm managed to outperform all the other methods in the reproduction of high-order statistics. Figures 22 and 23 reveal that the approach generates the smallest MAE between the simulated and the reference third and fourth-order cumulant maps for both magnification factors. Fine scale errors in the third and fourth-order cumulant maps are roughly one order of magnitude lower than the ones produced by other techniques.

Table 8 Summary statistics of the downscaled DEMs from the Jura Mountains example. Values are in residual elevation (in meters).

\begin{tabular}{lrrrrr}
\hline & $G$ & mean & std. dev. & min. & max. \\
\hline Training & 2 & -0.04 & 4.93 & -21.57 & 34.30 \\
Reference & 2 & -0.06 & 3.25 & -18.09 & 15.88 \\
Proposed method & 2 & $-0.06 \pm 0.00$ & $3.20 \pm 0.00$ & $-17.64 \pm 0.65$ & $15.93 \pm 0.70$ \\
Area-to-point & 2 & $-0.06 \pm 0.00$ & $3.42 \pm 0.01$ & $-17.87 \pm 0.38$ & $17.33 \pm 0.73$ \\
Direct sampling & 2 & $-0.06 \pm 0.00$ & $3.16 \pm 0.00$ & $-16.68 \pm 0.25$ & $16.59 \pm 0.90$ \\
Bicubic & 2 & -0.06 & 3.05 & -14.54 & 14.65 \\
\hline Training & 4 & -0.04 & 4.76 & -21.65 & 38.56 \\
Reference & 4 & -0.05 & 3.16 & -19.22 & 16.21 \\
Proposed method & 4 & $-0.05 \pm 0.00$ & $3.10 \pm 0.00$ & $-18.13 \pm 0.84$ & $16.97 \pm 0.83$ \\
Area-to-point & 4 & $-0.05 \pm 0.00$ & $3.34 \pm 0.01$ & $-18.18 \pm 0.37$ & $18.10 \pm 1.11$ \\
Direct sampling & 4 & $-0.05 \pm 0.00$ & $3.06 \pm 0.00$ & $-17.69 \pm 0.25$ & $16.86 \pm 1.00$ \\
Bicubic & 4 & -0.05 & 2.91 & -15.00 & 14.26 \\
\hline
\end{tabular}

\section{Discussion}

Results demonstrate that the proposed method is able to downscale coarse images with trends and reproduce target fine scale statistics. Simulations in both case studies are unbiased regarding conditioning to coarse resolution measurements. Fine scale topological properties such as the Euler characteristic and the probability of connec- 
Table 9 Validation of the Jura Mountains example.

\begin{tabular}{lrrrr}
\hline & $G$ & ME $(\mathrm{cm})$ & RMSE $(\mathrm{cm})$ & SSIM \\
\hline Proposed method & 2 & $-0.07 \pm 0.20$ & $21.75 \pm 0.39$ & $0.936 \pm 0.001$ \\
Area-to-point & 2 & $0.66 \pm 0.24$ & $18.32 \pm 0.59$ & $0.840 \pm 0.003$ \\
Direct sampling & 2 & $-0.42 \pm 0.37$ & $29.86 \pm 0.66$ & $0.914 \pm 0.002$ \\
Bicubic & 2 & 0.19 & 25.42 & 0.946 \\
\hline Proposed method & 4 & $0.00 \pm 0.21$ & $22.04 \pm 0.43$ & $0.906 \pm 0.001$ \\
Area-to-point & 4 & $0.62 \pm 0.25$ & $19.72 \pm 0.58$ & $0.803 \pm 0.003$ \\
Direct sampling & 4 & $-0.59 \pm 0.25$ & $23.73 \pm 0.27$ & $0.893 \pm 0.001$ \\
Bicubic & 4 & 0.24 & 29.94 & 0.917 \\
\hline
\end{tabular}

tion curves are also relatively well reproduced. Results also indicate good reproduction of second, third, and fourth-order statistics.

The exhaustive scanning of the training image for the $K$ best training data events allows the proposed framework to handle non-stationary data sets. This is often the case when one has to deal with non-constructed training images or simulate nonrepetitive structures. The distance-to-probability transformation function improves the reproduction of less frequent spatial structures and values by assigning higher conditional probabilities to the training data events that are more compatible with the local conditioning data. At the same time, it also allows the algorithm to generate variability on output realizations whenever multiple compatible data events are available in the training image. Building each local CPDF based upon the distanceto-probability transformation function is particularly important within the proposed iterative downscaling framework. Since fine scale features are conditionally simulated based on previously simulated data, the propagation of errors across scales can potentially compromise the simulation of finer resolution features. The framework is also particularly suitable for simulating textures that might contain both repetitive and non-repetitive structures. Conversely, traditional two-point statistics simulation methods infer the variogram model and histogram transformations using all available data. Therefore, they have trouble reproducing location-specific patterns and statistics. This also extends to MPS simulation algorithms which compute conditional probabilities based upon the entire training image. The management of non-stationary spatial patterns often requires the application of pre-processing routines prior to simulation (Boisvert et al. 2009 Boucher 2009a), which are not needed with the proposed approach.

Although the realizations globally honor the statistics and structural properties of the reference data, not all fine scale features can be recovered on the downscaled DEMs. Visually, it is noticeable that the texture of the realizations (Figs. 6 and 16) tends to be less sharp than the corresponding textures found on the reference fine resolution DEMs (Figs. 4 and 14). While the algorithm is to be able to generate realizations that depict the same type of variogram structures present in the reference fine resolution DEMs, simulations tend to underestimate the variability of the refer- 
ence data. This is a common problem for conditional MPS simulations. Straubhaar et al. (2016) reported the same phenomenon while running simulations constrained to block data, and Oriani et al. (2017) experienced a similar effect when simulating rainfall fields conditioned to weather state variables and DEMs. In our experiments, this effect is more evident when downscaling high-complexity terrains, such as the Western Alps example. One possible reason for this variance underestimation is that many of the structures to be recovered are significantly smaller than the pixel size of the coarse resolution image. In the super-resolution mapping literature, such scenario is classified as an L-resolution type problem (Atkinson 2009). Results indicate that some of these structures cannot be properly simulated when relying solely on coarse scale observations and previously simulated data. Imposed local conditioning constrains combined with the finite size of the training image may also play a role in preventing proper reproduction of such features. The addition of auxiliary fine scale covariates (e.g. high-resolution remote sensing imagery) might improve the simulation of these sub-pixel features. Further work is required to determine the magnification factor limits for different types of terrain and data sets.

A discussion about the criteria for selecting or constructing the dual-resolution training image is out of the scope of this paper. In geomorphological applications, the training image can be built from a better-informed analog data set. In other research areas, where analogs are not generally available, artificial training images might have to be employed. Inevitably, either analog-driven selection (Pérez et al. 2014) or artificial construction (Maharaja 2008) of training images will rely heavily on additional prior information based on expert knowledge. This information is fundamental when direct measurements of the fine resolution primary attribute are unavailable. For analog-derived training images, the selection process could be potentially guided by the coarse scale observations and indirect fine scale covariates (i.e. secondary data). Automated routines for training data selection grounded on exhaustive search over large training databases could be potentially implemented.

\section{Conclusions}

This paper presents a novel MPS simulation algorithm for downscaling images with trends. The method is illustrated with examples using DEMs from two geomorphologically distinct mountain ranges in Switzerland. Results show that the method is capable of generating fine resolution realizations that honor the input coarse resolution image and reproduce key structural properties and statistics.

To address the presence of trends in the data sets, the target variable is decomposed into a trend and a residual component at multiple scales. The trend component is downscaled with a deterministic interpolation method. The sub-pixel residual variable is simulated with a multi-scale sequential simulation framework. In order to improve the conditioning to coarse scale data, we propose the adoption of kernel weighting when computing the distances between target and training data events. 
We have introduced a new approach for integrating different support data in the context of distance-based MPS simulation. The proposed framework is well-suited for simulating images with non-repetitive structures, such as DEMs. The generality of the framework also offers the possibility to streamline the integration of other types of covariates. The transformation of distances between multivariate data events (with possibly different units or orders of magnitude) into probabilities facilitates the integration of multi-sensor data. The proposed scheme also eases the implementation of error/bias control systems (e.g. servo systems) (Remy et al. 2009) through direct manipulation of conditional probabilities.

Future work will explore the conflation of auxiliary variables to improve the quality and reduce the uncertainty associated with the downscaling process. The development of strategies to mitigate the generation of random noise on simulated realizations without causing loss of variability has particular importance for applications where the spatial structure of the downscaled image has an effect on the transfer function response. Particular effort will be put also on the development of an automated calibration procedure of the algorithm parameters based on a given training image. Additional research topics that should be investigated are the formulation of a quantitative criterion for selecting the training image, the evaluation of different distance-to-probability transformation functions and their impact on the structural quality and variability of simulated realizations, and the adaptation of the algorithm for supporting tridimensional data sets.

Acknowledgements The authors would like to thank Raphael Nussbaumer for providing the area-to-point simulation code and for the insightful discussions on adapting the approach for DEM downscaling. We are also grateful for the constructive comments of two anonymous reviewers which helped to improve the overall quality of the manuscript. This research was funded by the Swiss National Science Foundation, grant 200021_159756.

\section{References}

Allard D, Comunian A, Renard P (2012) Probability aggregation methods in geoscience. Mathematical Geosciences 44(5):545-581

Atkinson P M (2009) Issues of uncertainty in super-resolution mapping and their implications for the design of an inter-comparison study. International Journal of Remote Sensing 30(20):5293-5308

Atkinson P M (2013) Downscaling in remote sensing. International Journal of Applied Earth Observation and Geoinformation 22:106-114

Bertero M, Boccacci P (1998) Introduction to Inverse Problems in Imaging. IOP Publishing Ltd

Boisvert J B, Manchuk J G, Deutsch C V (2009) Kriging in the presence of locally varying anisotropy using non-Euclidean distances. Mathematical Geosciences 41(5):585-601

Boucher A (2009a) Considering complex training images with search tree partitioning. Computers \& Geosciences 35(6): 1151-1158

Boucher A (2009b) Sub-pixel mapping of coarse satellite remote sensing images with stochastic simulations from training images. Mathematical Geosciences 41(3):265-290

Boucher A, Kyriakidis P C (2007) Integrating fine scale information in super-resolution land-cover mapping. Photogrammetric Engineering \& Remote Sensing 73(8):913-921

Burt P, Adelson E (1983) The Laplacian pyramid as a compact image code. IEEE Transactions on Communications 31(4):532-540 
Dimitrakopoulos R, Mustapha H, Gloaguen E (2010) High-order statistics of spatial random fields: Exploring spatial cumulants for modeling complex non-Gaussian and non-linear phenomena. Mathematical Geosciences 42(1):65-99

Goovaerts P (1997) Geostatistics for Natural Resources Evaluation. Oxford University Press, New York

Guardiano F B, Srivastava R M (1993) Multivariate geostatistics: Beyond bivariate moments. In Quantitative Geology and Geostatistics, Springer Netherlands, 133-144

Hengl T, Bajat B, Blagojević D, Reuter H I (2008) Geostatistical modeling of topography using auxiliary maps. Computers \& Geosciences 34(12):1886-1899

Hoffimann J, Scheidt C, Barfod A, Caers J (2017) Stochastic simulation by image quilting of process-based geological models. Computers \& Geosciences 106:18-32

Journel A G (1999) Markov models for cross-covariances. Mathematical Geology 31(8):955-964

Journel A G, Deutsch C V (1993) Entropy and spatial disorder. Mathematical Geology 25(3):329-355

Journel A G, Huijbregts C J (1978) Mining Geostatistics. Academic Press, New York

Journel A G, Rossi M E (1989) When do we need a trend model in kriging? Mathematical Geology 21(7):715-739

Keys R (1981) Cubic convolution interpolation for digital image processing. IEEE Transactions on Acoustics, Speech, and Signal Processing 29(6):1153-1160

Kwatra V, Schödl A, Essa I, Turk G, Bobick A (2003) Graphcut textures: Image and video synthesis using graph cuts. ACM Transactions on Graphics 22(3):277-286

Kyriakidis P C, Shortridge A M, Goodchild M F (1999) Geostatistics for conflation and accuracy assessment of digital elevation models. International Journal of Geographical Information Science 13(7):677707

Kyriakidis P C, Yoo E H (2005) Geostatistical prediction and simulation of point values from areal data. Geographical Analysis 37(2):124-151

Liu Y, Journel A G (2009) A package for geostatistical integration of coarse and fine scale data. Computers \& Geosciences 35(3):527-547

Maharaja A (2008) TiGenerator: Object-based training image generator. Computers \& Geosciences 34(12):1753-1761

Mariethoz G, Renard P, Straubhaar J (2010) The direct sampling method to perform multiple-point geostatistical simulations. Water Resources Research 46(11)

Mariethoz G, Renard P, Straubhaar J (2011) Extrapolating the fractal characteristics of an image using scale-invariant multiple-point statistics. Mathematical Geosciences 43(7):783-797

Meerschman E, Pirot G, Mariethoz G, Straubhaar J, Meirvenne M V, Renard P (2013) A practical guide to performing multiple-point statistical simulations with the direct sampling algorithm. Computers \& Geosciences 52:307-324

Oriani F, Ohana-Levi N, Marra F, Straubhaar J, Mariethoz G, Renard P, Karnieli A, Morin E (2017) Simulating small-scale rainfall fields conditioned by weather state and elevation: A data-driven approach based on rainfall radar images. Water Resources Research 53(10):8512-8532

Pérez C, Mariethoz G, Ortiz J M (2014) Verifying the high-order consistency of training images with data for multiple-point geostatistics. Computers \& Geosciences 70:190-205

Ravalec M L, Noetinger B, Hu L Y (2000) The FFT moving average (FFT-MA) generator: An efficient numerical method for generating and conditioning Gaussian simulations. Mathematical Geology 32(6):701-723

Remy N, Boucher A, Wu J (2009) Applied Geostatistics with SGeMS: A User's Guide. Cambridge University Press

Renard P, Allard D (2013) Connectivity metrics for subsurface flow and transport. Advances in Water Resources 51:168-196

Straubhaar J, Renard P, Mariethoz G (2016) Conditioning multiple-point statistics simulations to block data. Spatial Statistics 16:53-71

Strebelle S (2002) Conditional simulation of complex geological structures using multiple-point statistics. Mathematical Geology 34(1):1-21

Tang Y, Zhang J, Jing L, Li H (2015) Digital elevation data fusion using multiple-point geostatistical simulation. IEEE Journal of Selected Topics in Applied Earth Observations and Remote Sensing 8(10):4922-4934

Tran T T (1994) Improving variogram reproduction on dense simulation grids. Computers \& Geosciences 20(7-8):1161-1168

Wang Z, Bovik A, Sheikh H, Simoncelli E (2004) Image quality assessment: From error visibility to structural similarity. IEEE Transactions on Image Processing 13(4):600-612 
Wiederkehr M, Möri A (2013) SwissALTI3D: A new tool for geological mapping. Bulletin fuer Angewandte Geologie 18(1):61-69

Zagayevskiy Y, Deutsch C V (2015) Multivariate grid-free geostatistical simulation with point or block scale secondary data. Stochastic Environmental Research and Risk Assessment 30(6):1613-1633

Zhang T, Switzer P, Journel A (2006) Filter-based classification of training image patterns for spatial simulation. Mathematical Geology 38(1):63-80 

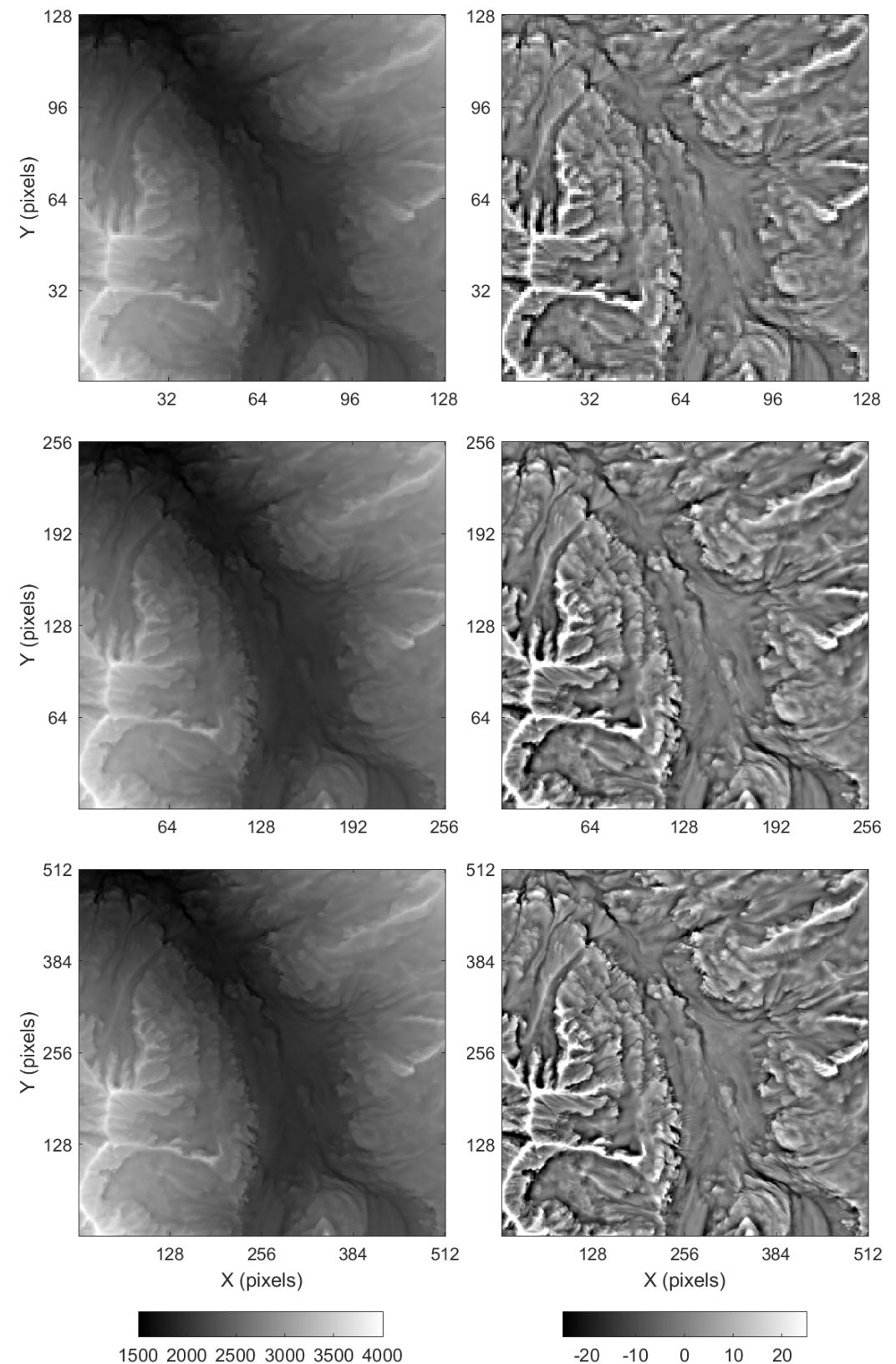

Fig. 5 The Western Alps training DEMs. Top left: coarse resolution DEM. Top right: coarse resolution residual DEM. Middle left: medium resolution DEM. Middle right: medium resolution residual DEM. Bottom left: fine resolution DEM. Bottom right: fine resolution residual DEM. The residual component of the trended DEMs has a vertical exaggeration factor of $8 \mathrm{x}$. Colorbars' unit is meter. 

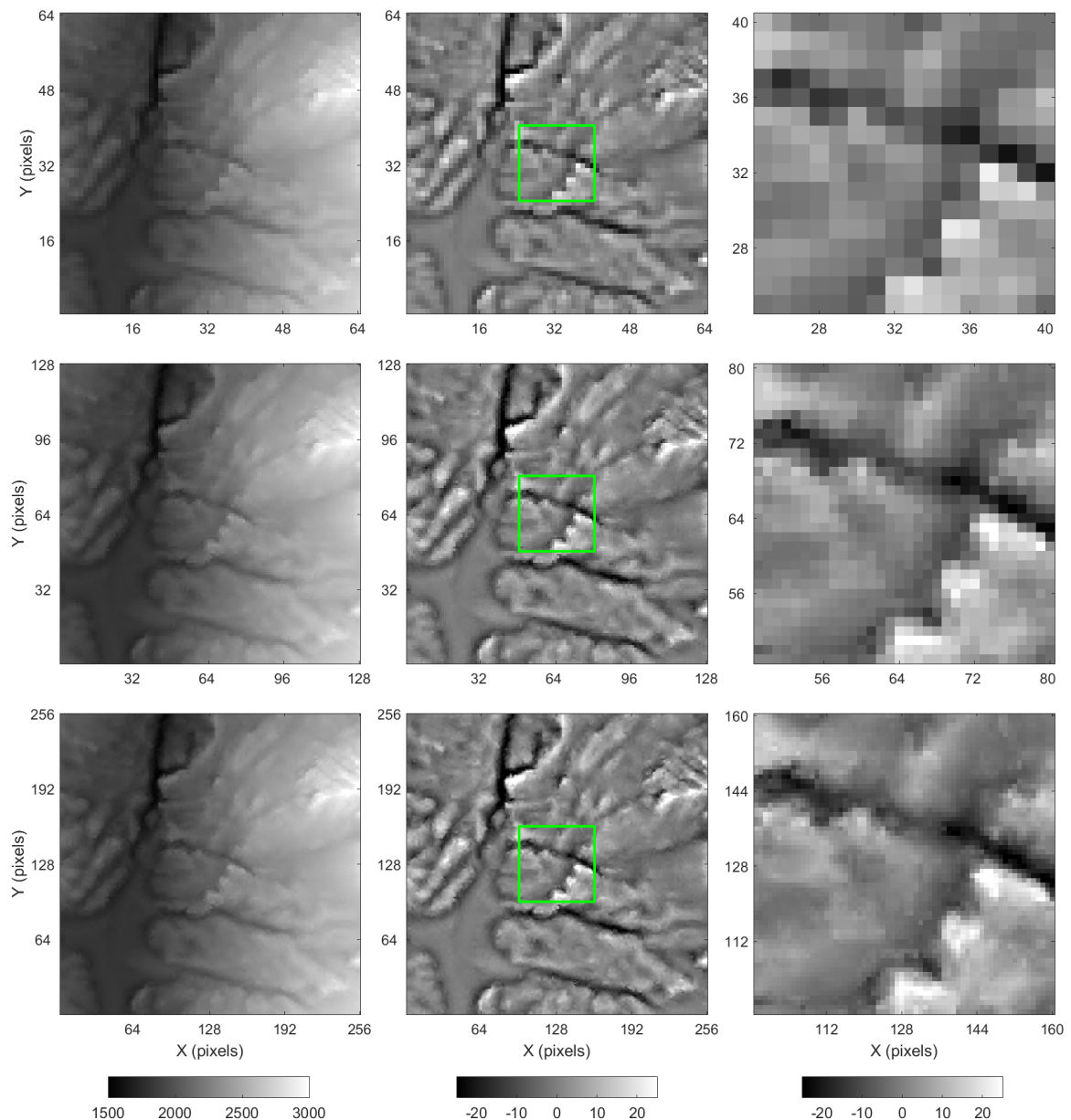

Fig. 6 The Western Alps downscaled DEMs produced with the proposed algorithm. Top left: coarse resolution DEM. Top center: coarse resolution residual DEM. Top right: coarse resolution zoom. Middle left simulated medium resolution DEM. Middle center: simulated medium resolution residual DEM. Middle right: medium resolution zoom. Bottom left: simulated fine resolution DEM. Bottom center: simulated fine resolution residual DEM. Bottom right: fine resolution zoom. The residual component of the trended DEMs has a vertical exaggeration factor of $8 \mathrm{x}$. Green boxes highlight the magnified area. Colorbars' unit is meter. 

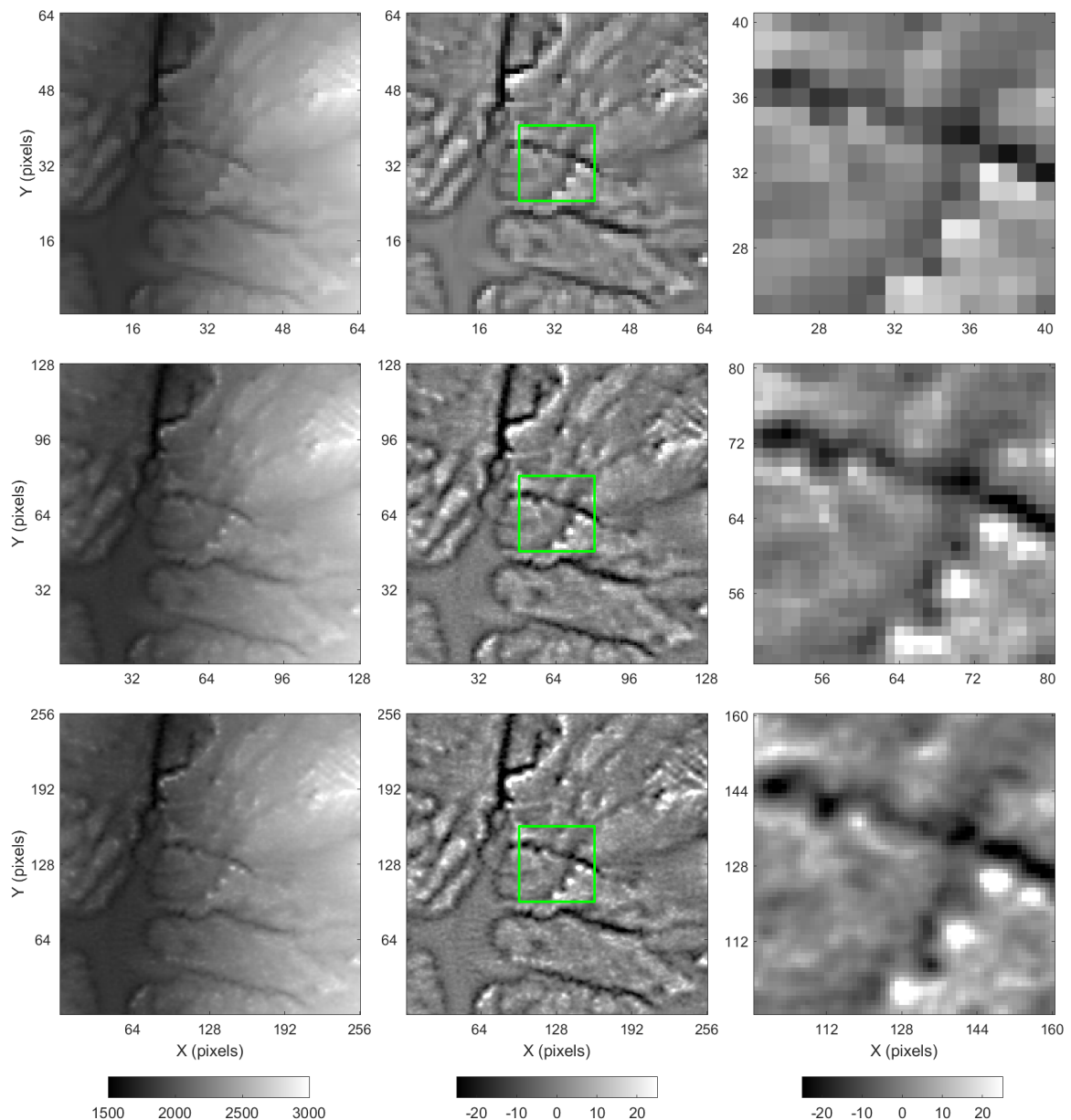

Fig. 7 The Western Alps downscaled DEMs produced with area-to-point simulation. Top left: coarse resolution DEM. Top center: coarse resolution residual DEM. Top right: coarse resolution zoom. Middle left: simulated medium resolution DEM. Middle center: simulated medium resolution residual DEM. Middle right: medium resolution zoom. Bottom left: simulated fine resolution DEM. Bottom center: simulated fine resolution residual DEM. Bottom right: fine resolution zoom. The residual component of the trended DEMs has a vertical exaggeration factor of $8 x$. Green boxes highlight the magnified area. Colorbars' unit is meter. 

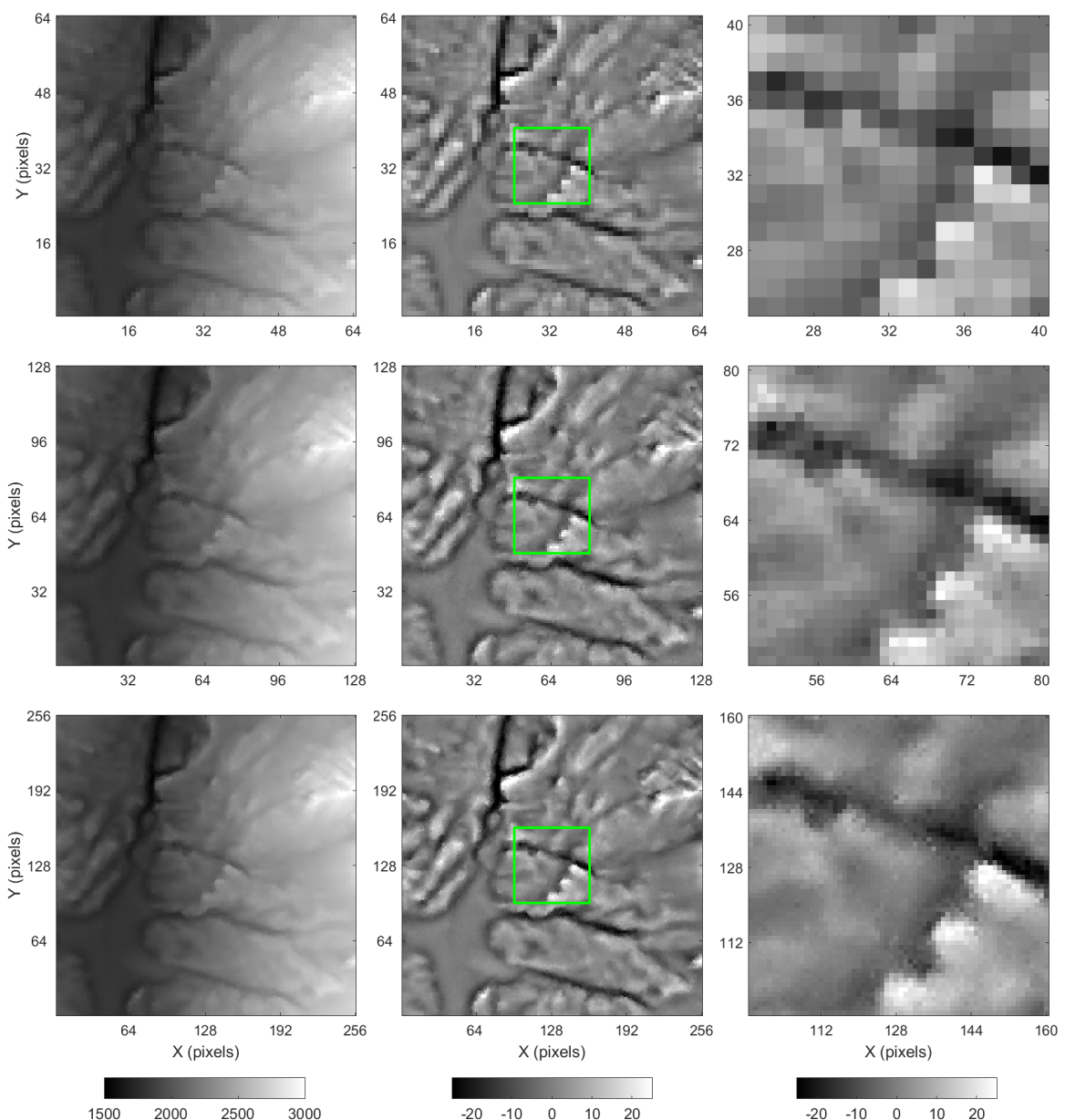

Fig. 8 The Western Alps downscaled DEMs produced with direct sampling. Top left: coarse resolution DEM. Top center: coarse resolution residual DEM. Top right: coarse resolution zoom. Middle left: simulated medium resolution DEM. Middle center: simulated medium resolution residual DEM. Middle right: medium resolution zoom. Bottom left: simulated fine resolution DEM. Bottom center: simulated fine resolution residual DEM. Bottom right: fine resolution zoom. The residual component of the trended DEMs has a vertical exaggeration factor of $8 \mathrm{x}$. Green boxes highlight the magnified area. Colorbars' unit is meter. 

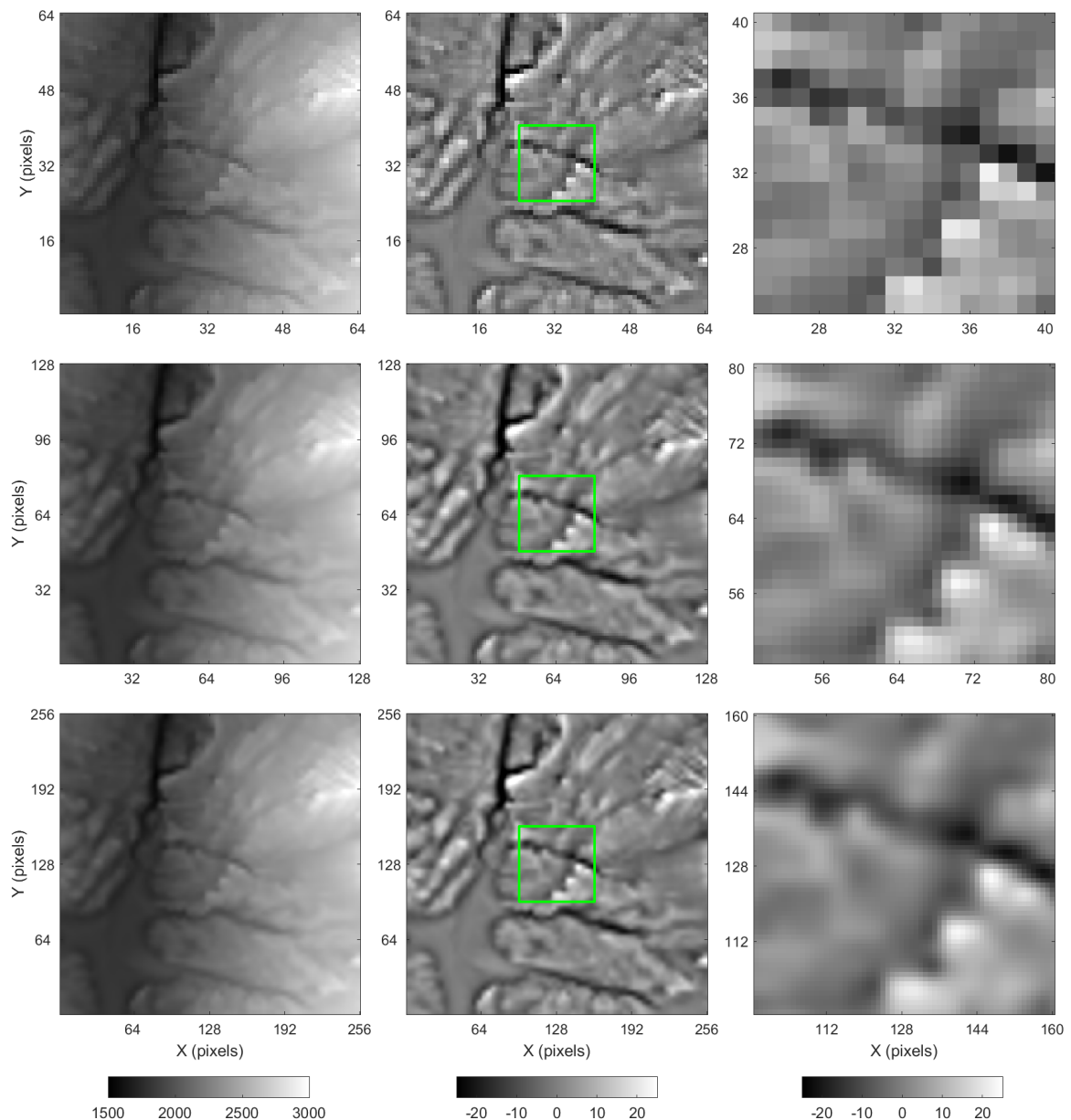

Fig. 9 The Western Alps downscaled DEMs produced with bicubic interpolation. Top left: coarse resolution DEM. Top center: coarse resolution residual DEM. Top right: coarse resolution zoom. Middle left: interpolated medium resolution DEM. Middle center: interpolated medium resolution residual DEM. Middle right: medium resolution zoom. Bottom left: interpolated fine resolution DEM. Bottom center: interpolated fine resolution residual DEM. Bottom right: fine resolution zoom. The residual component of the trended DEMs has a vertical exaggeration factor of 8x. Green boxes highlight the magnified area. Colorbars' unit is meter. 

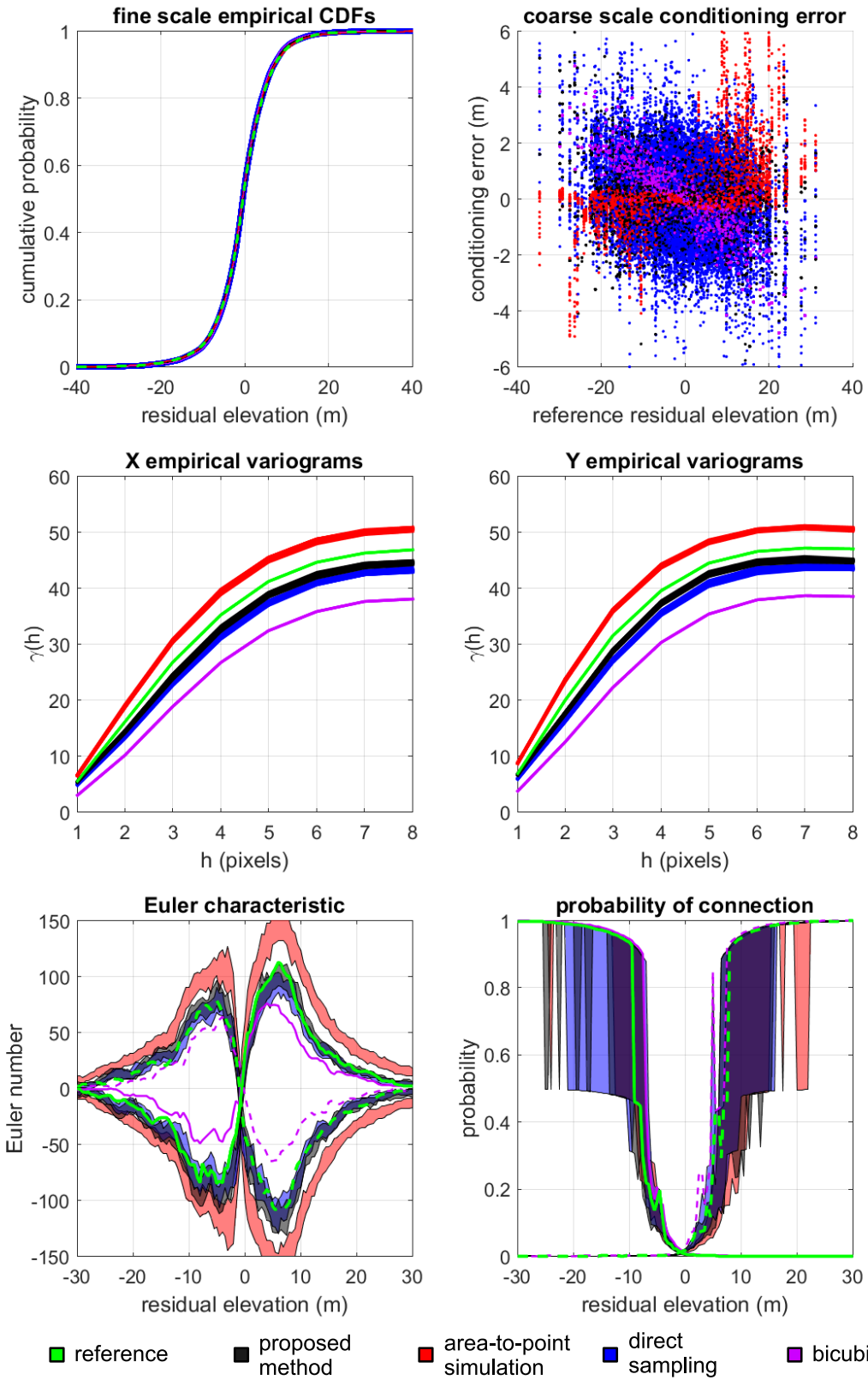

$$
\square \begin{aligned}
& \text { area-to-point } \\
& \text { simulation }
\end{aligned}
$$

$\square$ bicubic

Fig. 10 Validation of the Western Alps example $(G=2)$. Top left: sub-pixel empirical CDFs. Top right: scatter plots between reference coarse residual elevation and conditioning error. Middle left: sub-pixel empirical variograms along the $\mathrm{X}$ axis. Middle right: sub-pixel empirical variograms along the $\mathrm{Y}$ axis. Bottom left: fine scale Euler characteristic. Bottom right: fine scale probability of connection. 

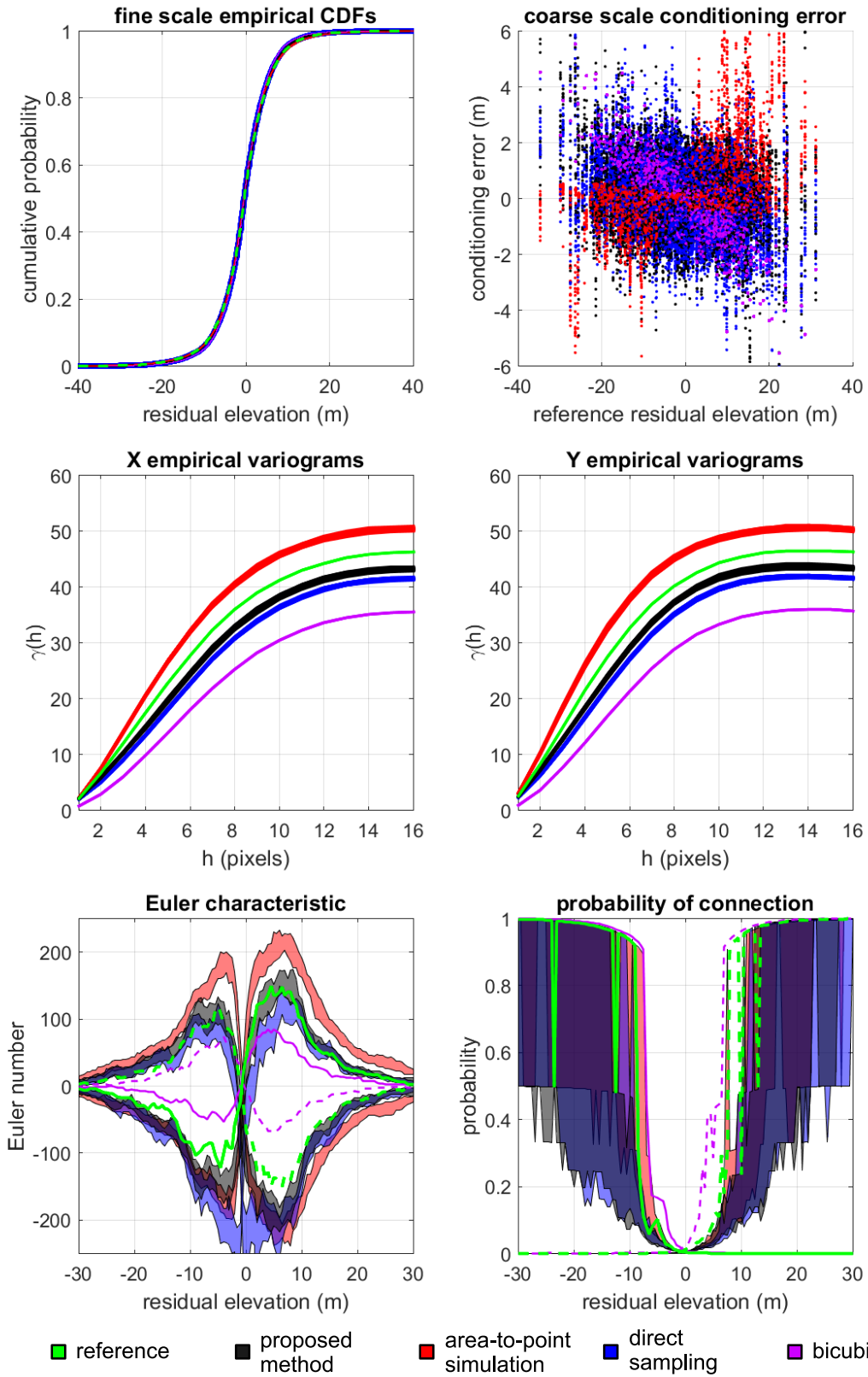

$\square \begin{aligned} & \text { area-to-point } \\ & \text { simulation }\end{aligned} \begin{aligned} & \text { direct } \\ & \text { sampling }\end{aligned}$

$\square$ bicubic

Fig. 11 Validation of the Western Alps example $(G=4)$. Top left: sub-pixel empirical CDFs. Top right: scatter plots between reference coarse residual elevation and conditioning error. Middle left: sub-pixel empirical variograms along the $\mathrm{X}$ axis. Middle right: sub-pixel empirical variograms along the $\mathrm{Y}$ axis. Bottom left: fine scale Euler characteristic. Bottom right: fine scale probability of connection. 


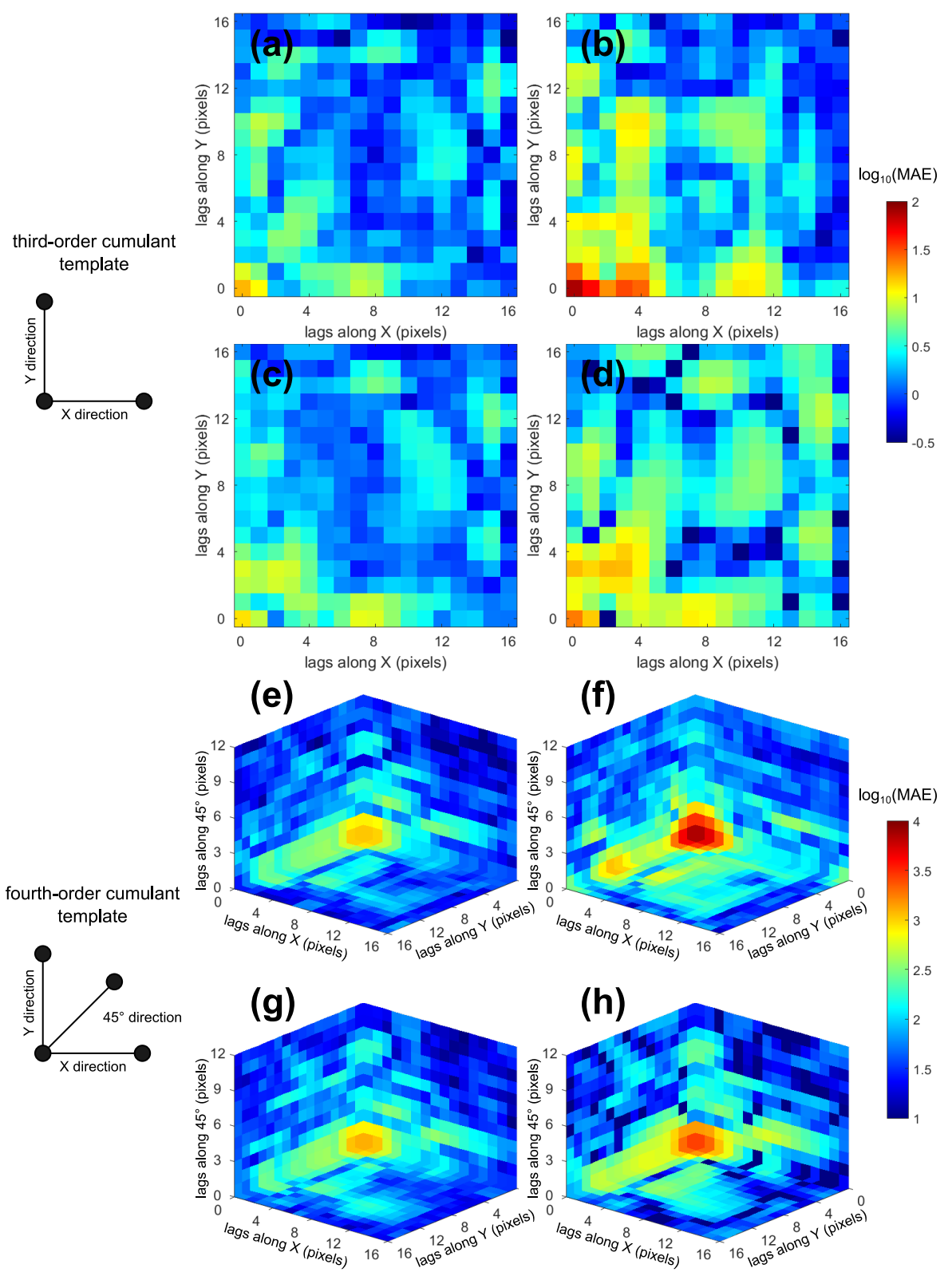

Fig. 12 MAE between simulated and reference third and fourth-order cumulant maps from the Western Alps example $(G=2)$. Top: third-order cumulant MAE maps for a proposed method, b area-to-point simulation, $\mathbf{c}$ direct sampling, and $\mathbf{d}$ bicubic interpolation. Colorbar unit is $\mathrm{m}^{3}$. Bottom: fourth-order cumulant MAE maps for $\mathbf{e}$ proposed method, $\mathbf{f}$ area-to-point simulation, $\mathbf{g}$ direct sampling, and $\mathbf{h}$ bicubic interpolation. Colorbar unit is $\mathrm{m}^{4}$. 


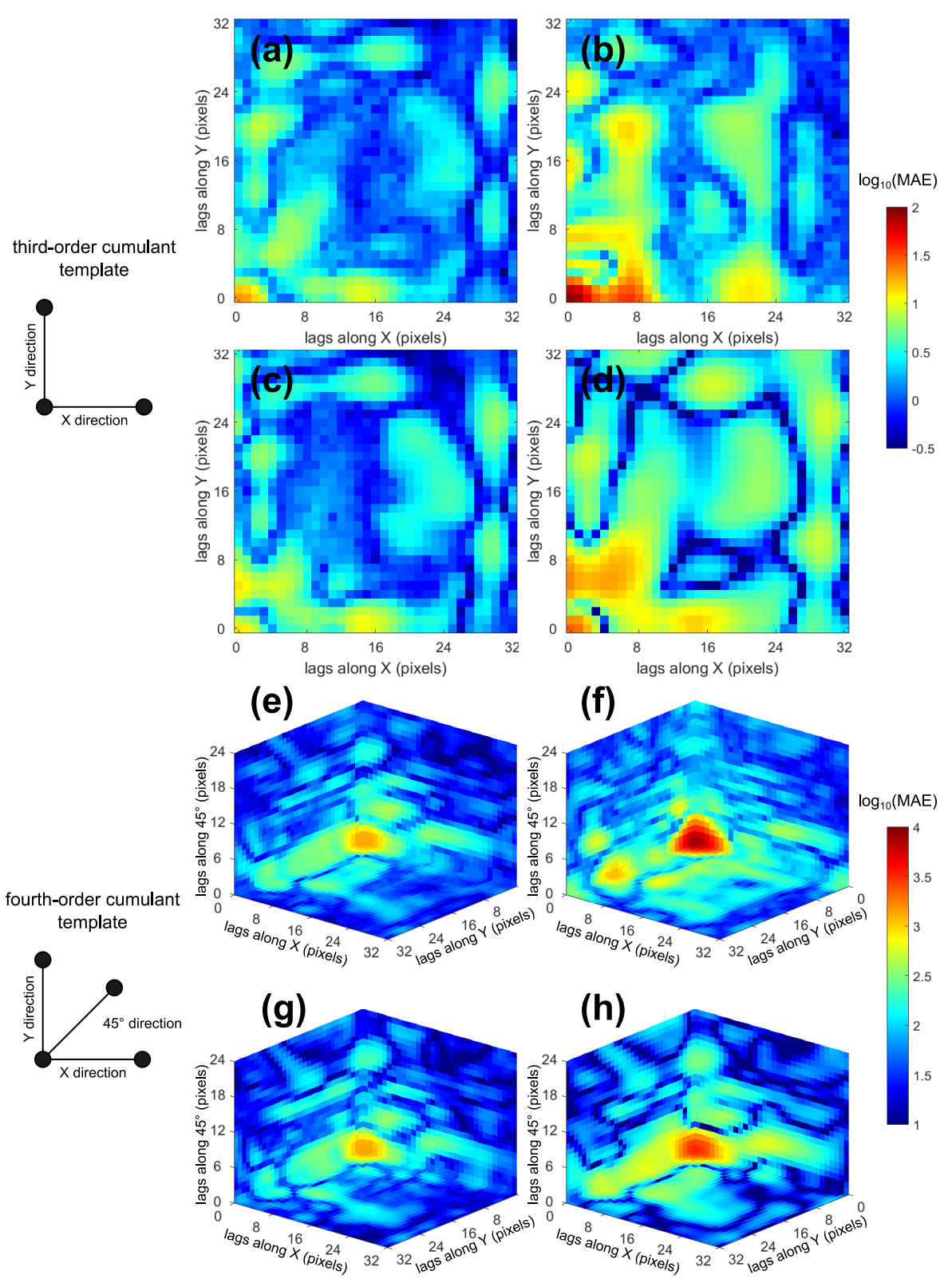

Fig. 13 MAE between simulated and reference third and fourth-order cumulant maps from the Western Alps example $(G=4)$. Top: third-order cumulant MAE maps for a proposed method, b area-to-point simulation, c direct sampling, and $\mathbf{d}$ bicubic interpolation. Colorbar unit is $\mathrm{m}^{3}$. Bottom: fourth-order cumulant MAE maps for $\mathbf{e}$ proposed method, $\mathbf{f}$ area-to-point simulation, $\mathbf{g}$ direct sampling, and $\mathbf{h}$ bicubic interpolation. Colorbar unit is $\mathrm{m}^{4}$. 

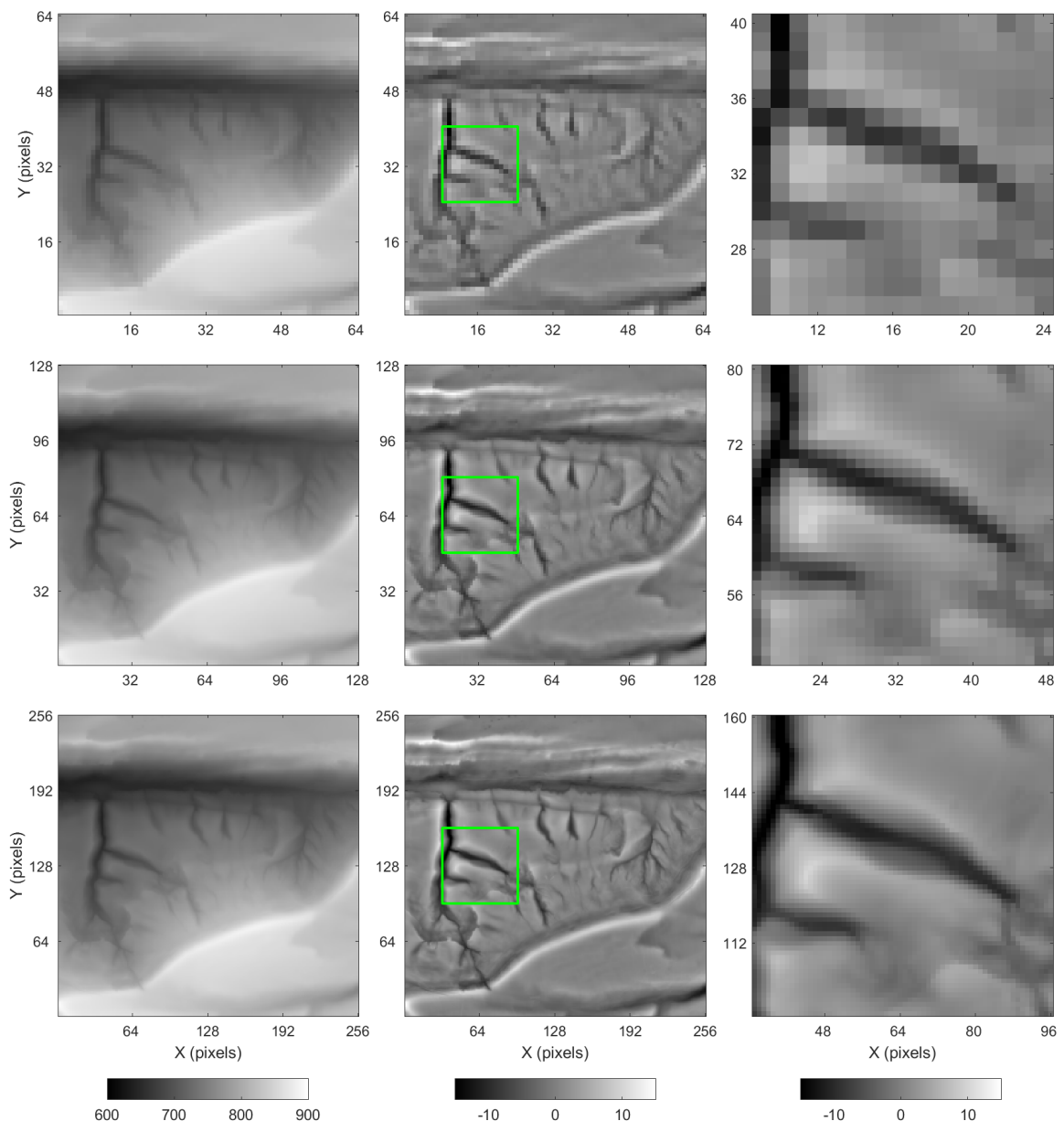

Fig. 14 The Jura Mountains reference DEMs. Top left: coarse resolution DEM. Top center: coarse resolution residual DEM. Top right: coarse resolution zoom. Middle left: medium resolution DEM. Middle center: medium resolution residual DEM. Middle right: medium resolution zoom. Bottom left: fine resolution DEM. Bottom center: fine resolution residual DEM. Bottom right: fine resolution zoom. The residual component of the trended DEMs has a vertical exaggeration factor of 2x. Green boxes highlight the magnified area. Colorbars' unit is meter. 

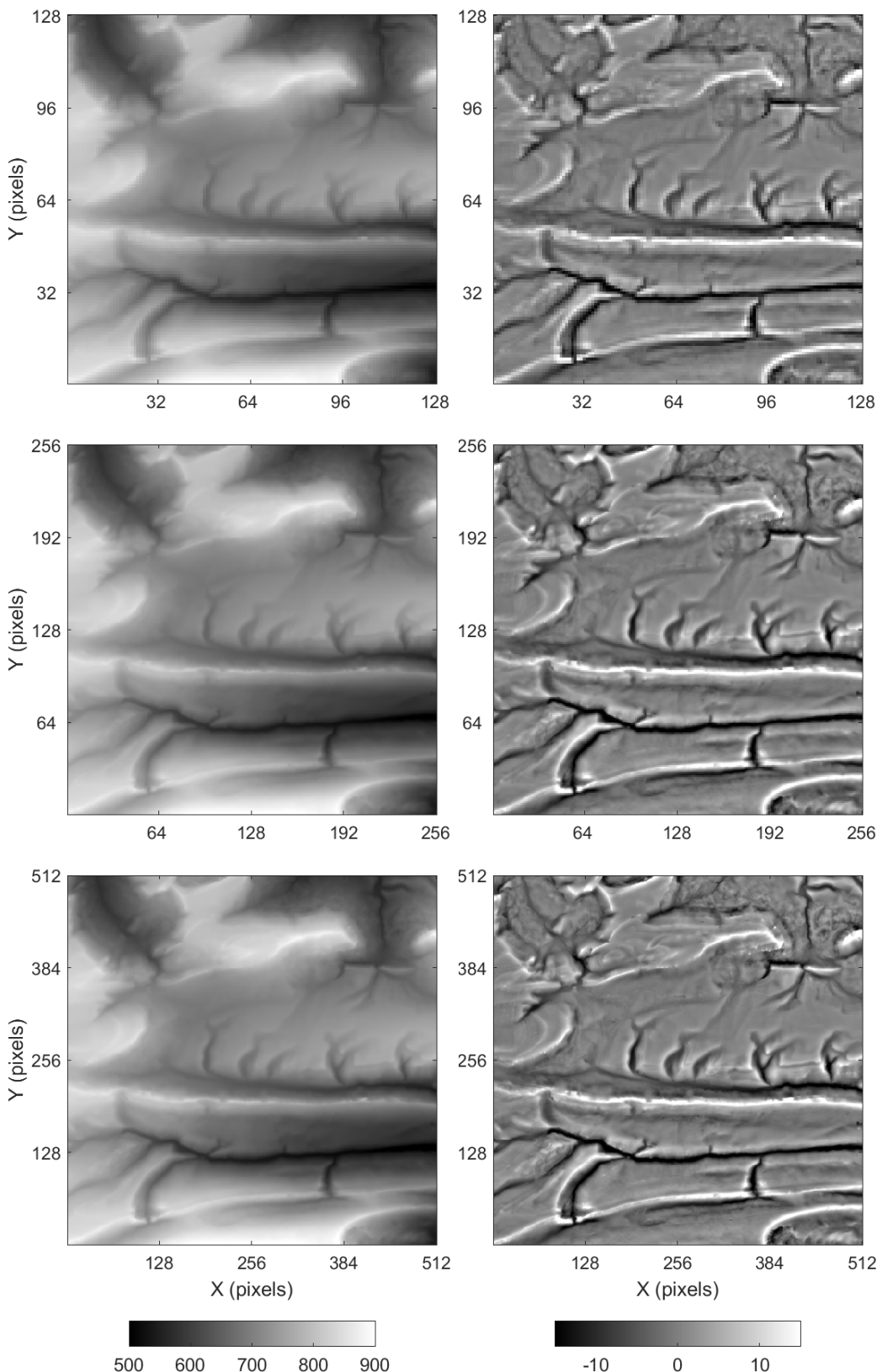

Fig. 15 The Jura Mountains training DEMs. Top left: coarse resolution DEM with trend. Top right: coarse resolution residual DEM. Middle left: medium resolution DEM with trend. Middle right: medium resolution residual DEM. Bottom left: fine resolution DEM with trend. Bottom right: fine resolution residual DEM. The residual component of the trended DEMs has a vertical exaggeration factor of $2 \mathrm{x}$. Colorbars' unit is meter. 

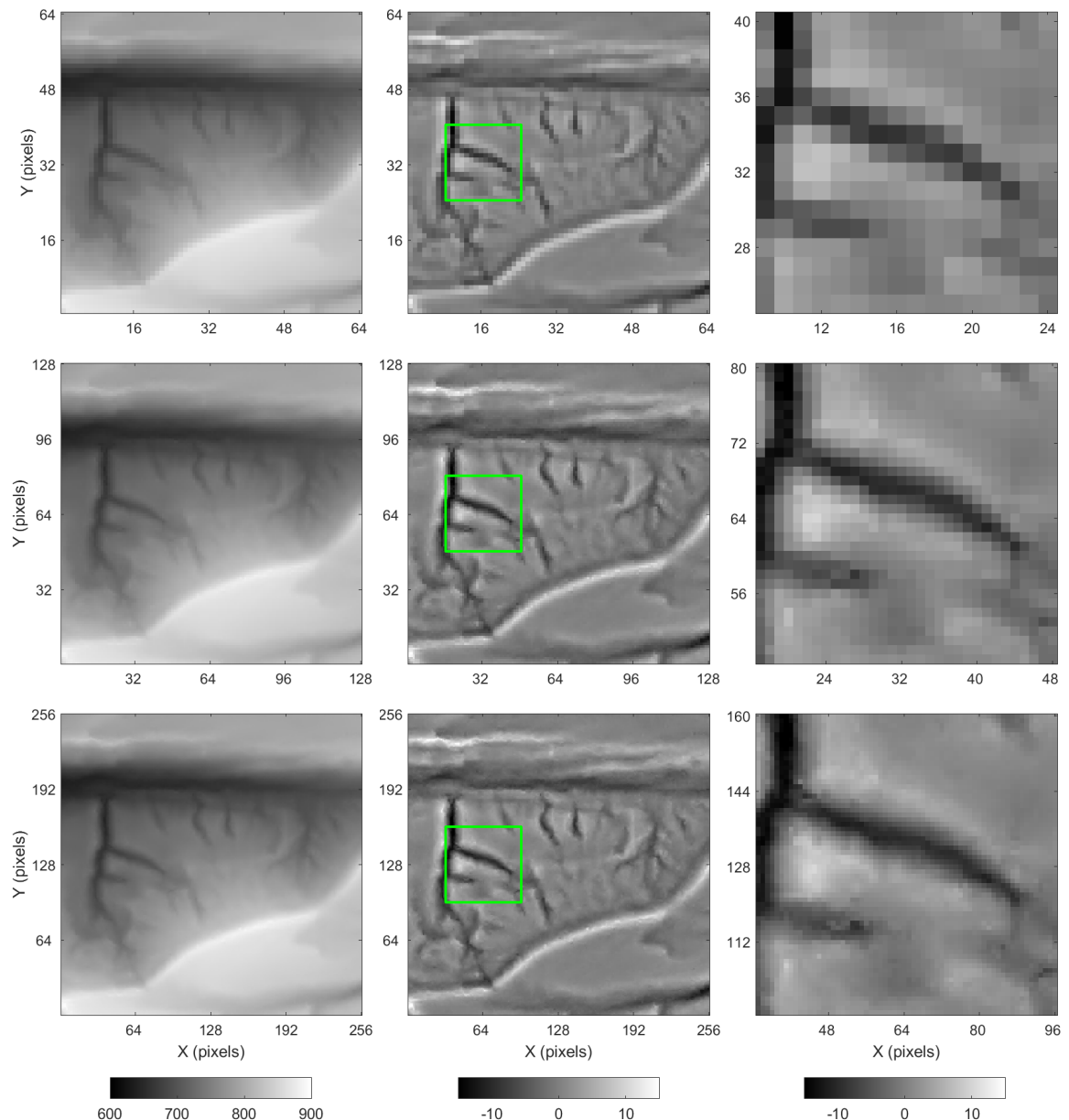

Fig. 16 The Jura Mountains downscaled DEMs produced with the proposed algorithm. Top left: coarse resolution DEM. Top center: coarse resolution residual DEM. Top right: coarse resolution zoom. Middle left: simulated medium resolution DEM. Middle center: simulated medium resolution residual DEM. Middle right: medium resolution zoom. Bottom left: simulated fine resolution DEM. Bottom center: simulated fine resolution residual DEM. Bottom right: fine resolution zoom. The residual component of the trended DEMs has a vertical exaggeration factor of $2 x$. Green boxes highlight the magnified area. Colorbars' unit is meter. 

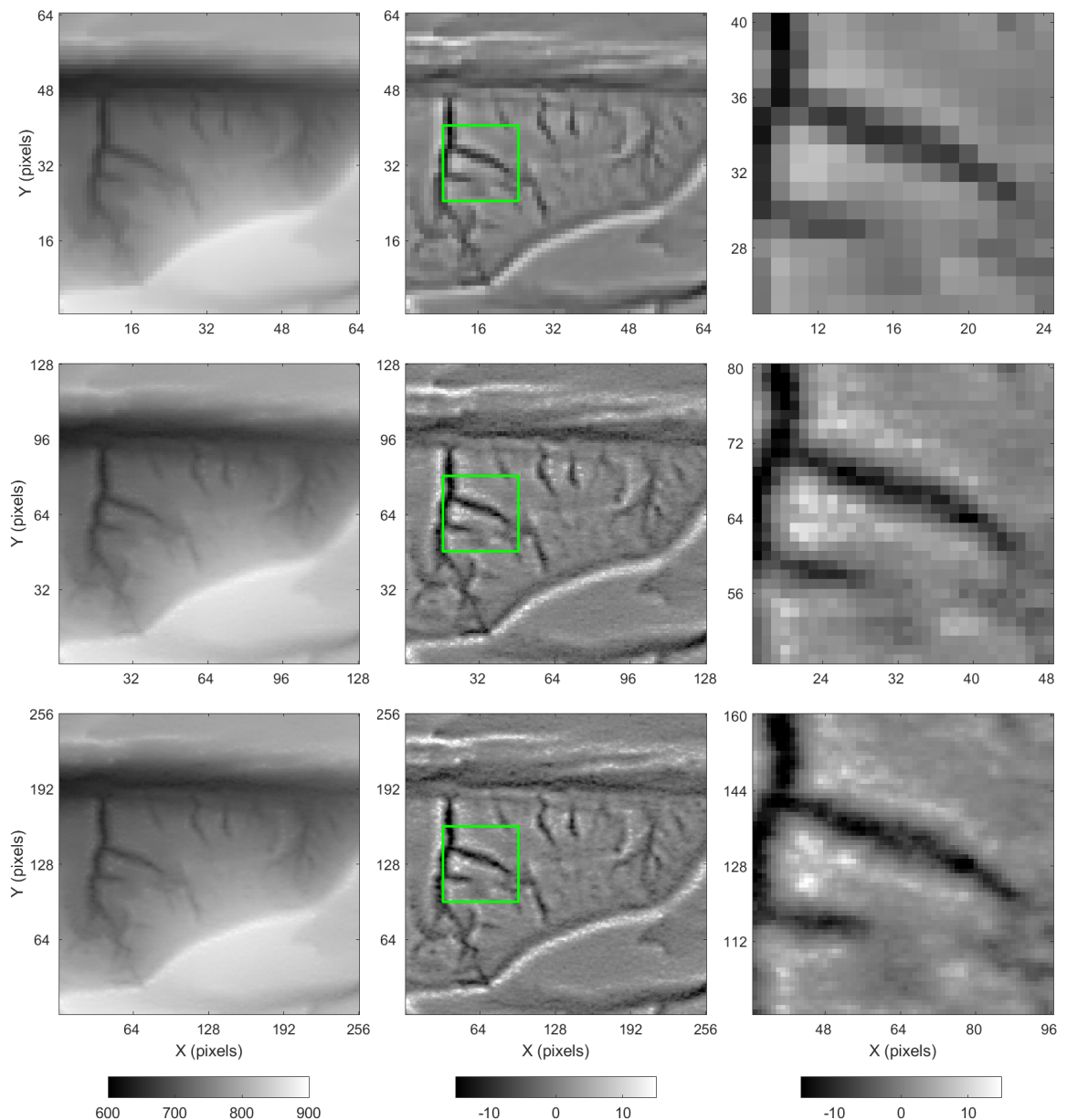

Fig. 17 The Jura Mountains downscaled DEMs produced with area-to-point simulation. Top left: coarse resolution DEM. Top center: coarse resolution residual DEM. Top right: coarse resolution zoom. Middle left: simulated medium resolution DEM. Middle center: simulated medium resolution residual DEM. Middle right: medium resolution zoom. Bottom left: simulated fine resolution DEM. Bottom center: simulated fine resolution residual DEM. Bottom right: fine resolution zoom. The residual component of the trended DEMs has a vertical exaggeration factor of 2x. Green boxes highlight the magnified area. Colorbars' unit is meter. 

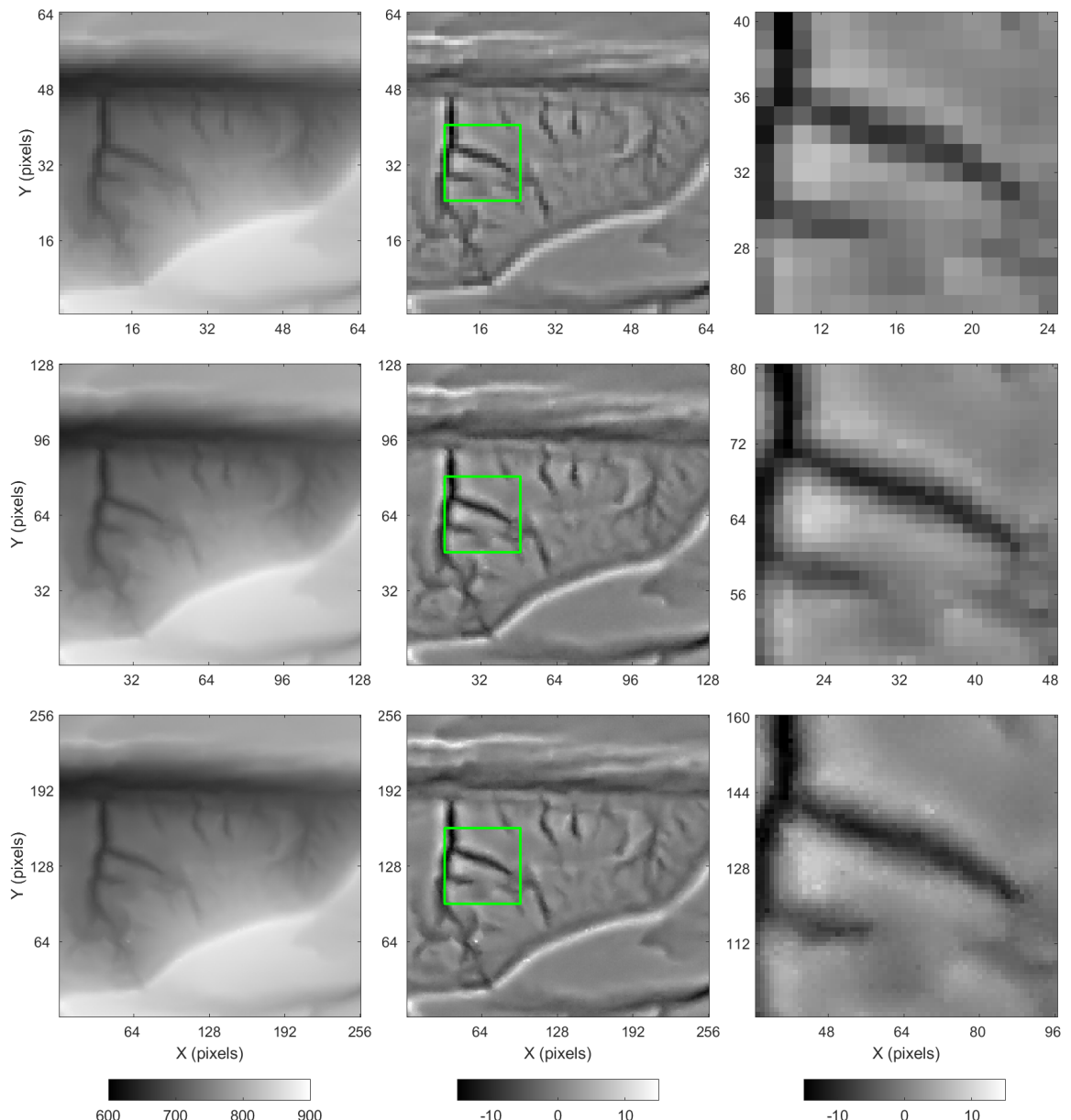

Fig. 18 The Jura Mountains downscaled DEMs produced with direct sampling. Top left: coarse resolution DEM. Top center: coarse resolution residual DEM. Top right: coarse resolution zoom. Middle left: simulated medium resolution DEM. Middle center: simulated medium resolution residual DEM. Middle right: medium resolution zoom. Bottom left: simulated fine resolution DEM. Bottom center: simulated fine resolution residual DEM. Bottom right: fine resolution zoom. The residual component of the trended DEMs has a vertical exaggeration factor of $2 x$. Green boxes highlight the magnified area. Colorbars' unit is meter. 

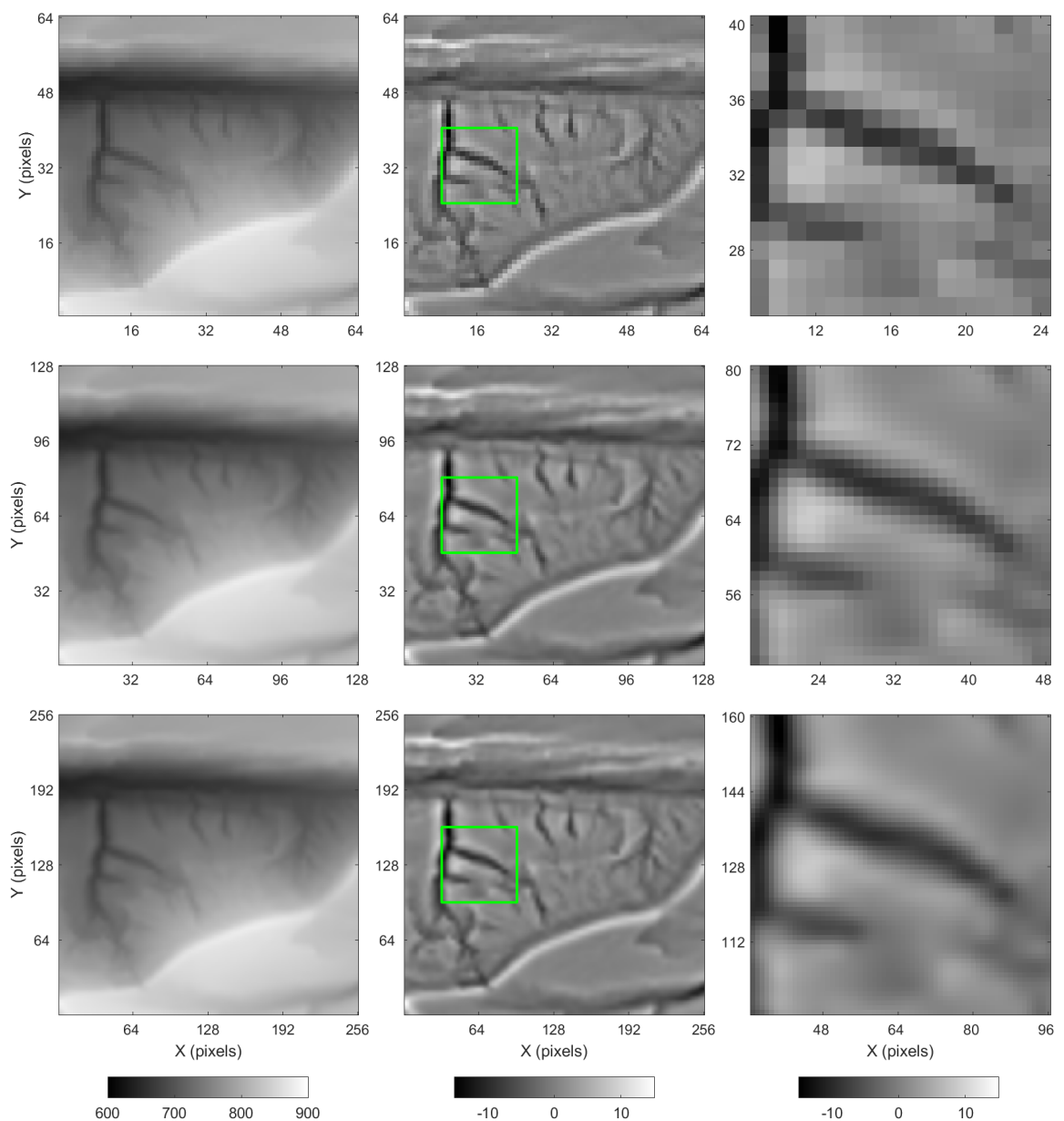

Fig. 19 The Jura Mountains downscaled DEMs produced with bicubic interpolation. Top left: coarse resolution DEM. Top center: coarse resolution residual DEM. Top right: coarse resolution zoom. Middle left: interpolated medium resolution DEM. Middle center: interpolated medium resolution residual DEM. Middle right: medium resolution zoom. Bottom left: interpolated fine resolution DEM. Bottom center: interpolated fine resolution residual DEM. Bottom right: fine resolution zoom. The residual component of the trended DEMs has a vertical exaggeration factor of 2x. Green boxes highlight the magnified area. Colorbars' unit is meter. 

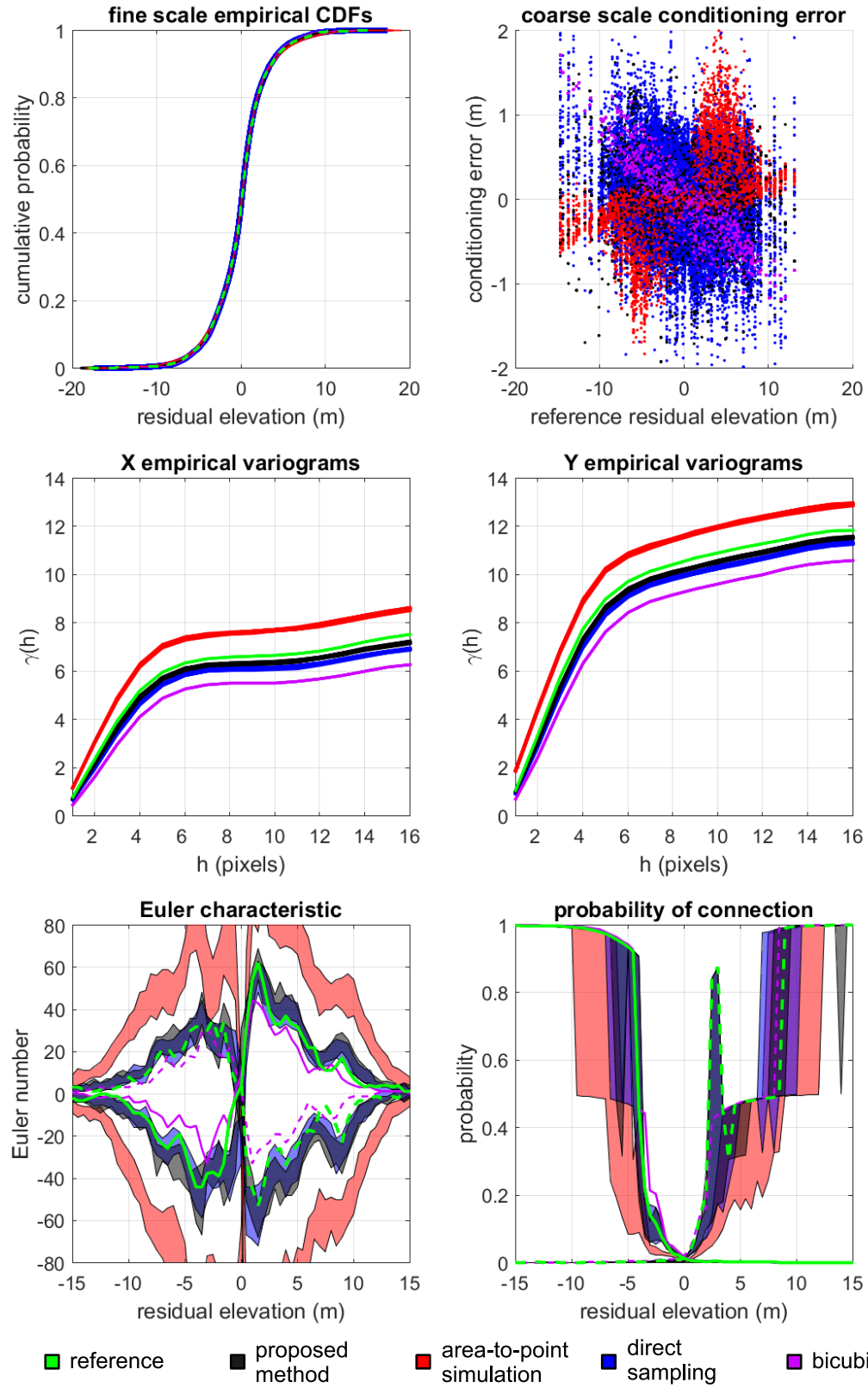

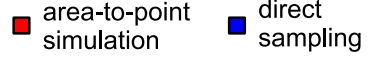

$\square$ bicubic

Fig. 20 Validation of the Jura Mountains example $(G=2)$. Top left: sub-pixel empirical CDFs. Top right: scatter plots between reference coarse residual elevation and conditioning error. Middle left: sub-pixel empirical variograms along the $\mathrm{X}$ axis. Middle right: sub-pixel empirical variograms along the $\mathrm{Y}$ axis. Bottom left: fine scale Euler characteristic. Bottom right: fine scale probability of connection. 

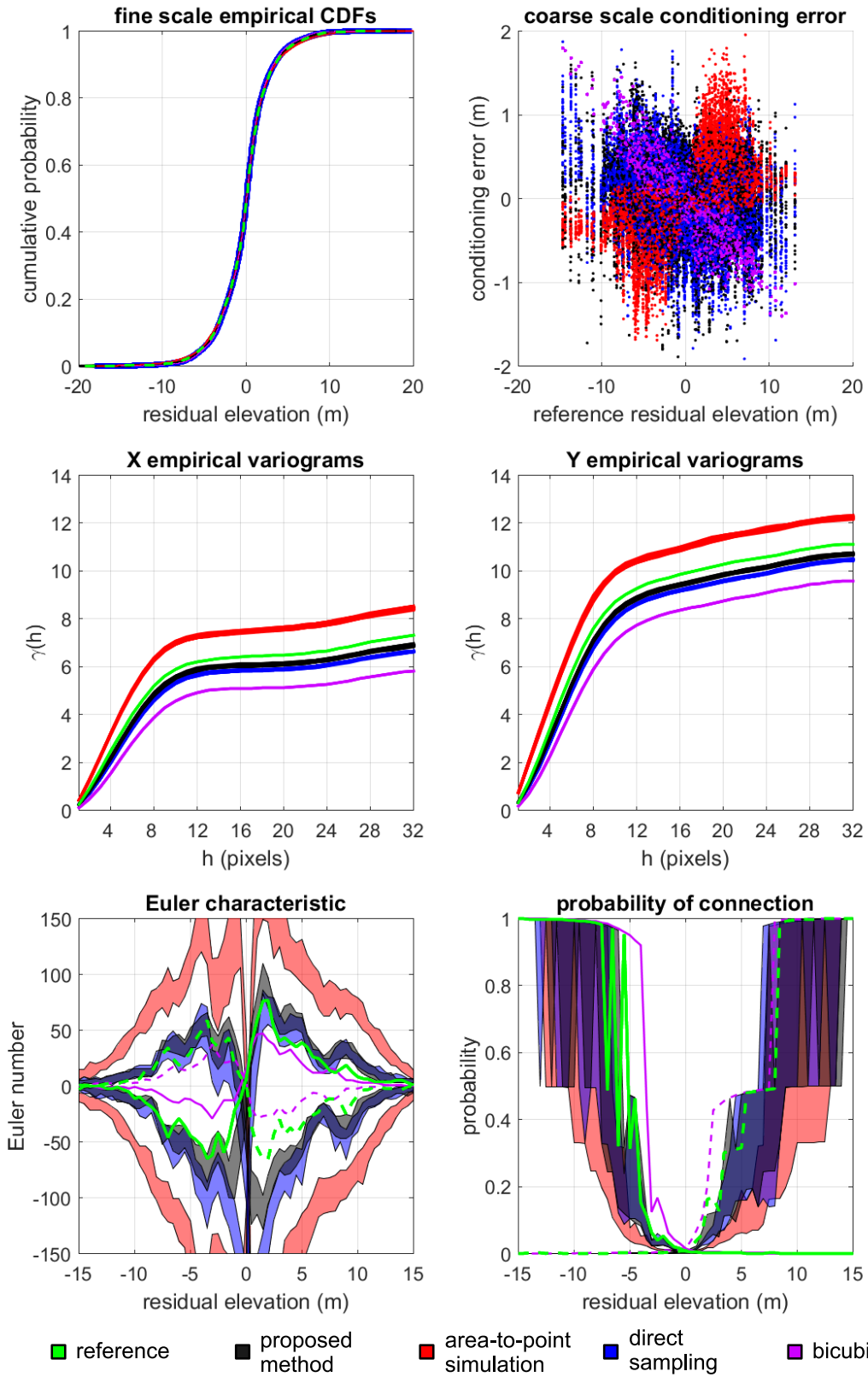

$\square \begin{aligned} & \text { area-to-point } \\ & \text { simulation }\end{aligned} \begin{aligned} & \text { direct } \\ & \text { sampling }\end{aligned}$

$\square$ bicubic

Fig. 21 Validation of the Jura Mountains example $(G=4)$. Top left: sub-pixel empirical CDFs. Top right: scatter plots between reference coarse residual elevation and conditioning error. Middle left: sub-pixel empirical variograms along the $\mathrm{X}$ axis. Middle right: sub-pixel empirical variograms along the $\mathrm{Y}$ axis. Bottom left: fine scale Euler characteristic. Bottom right: fine scale probability of connection. 


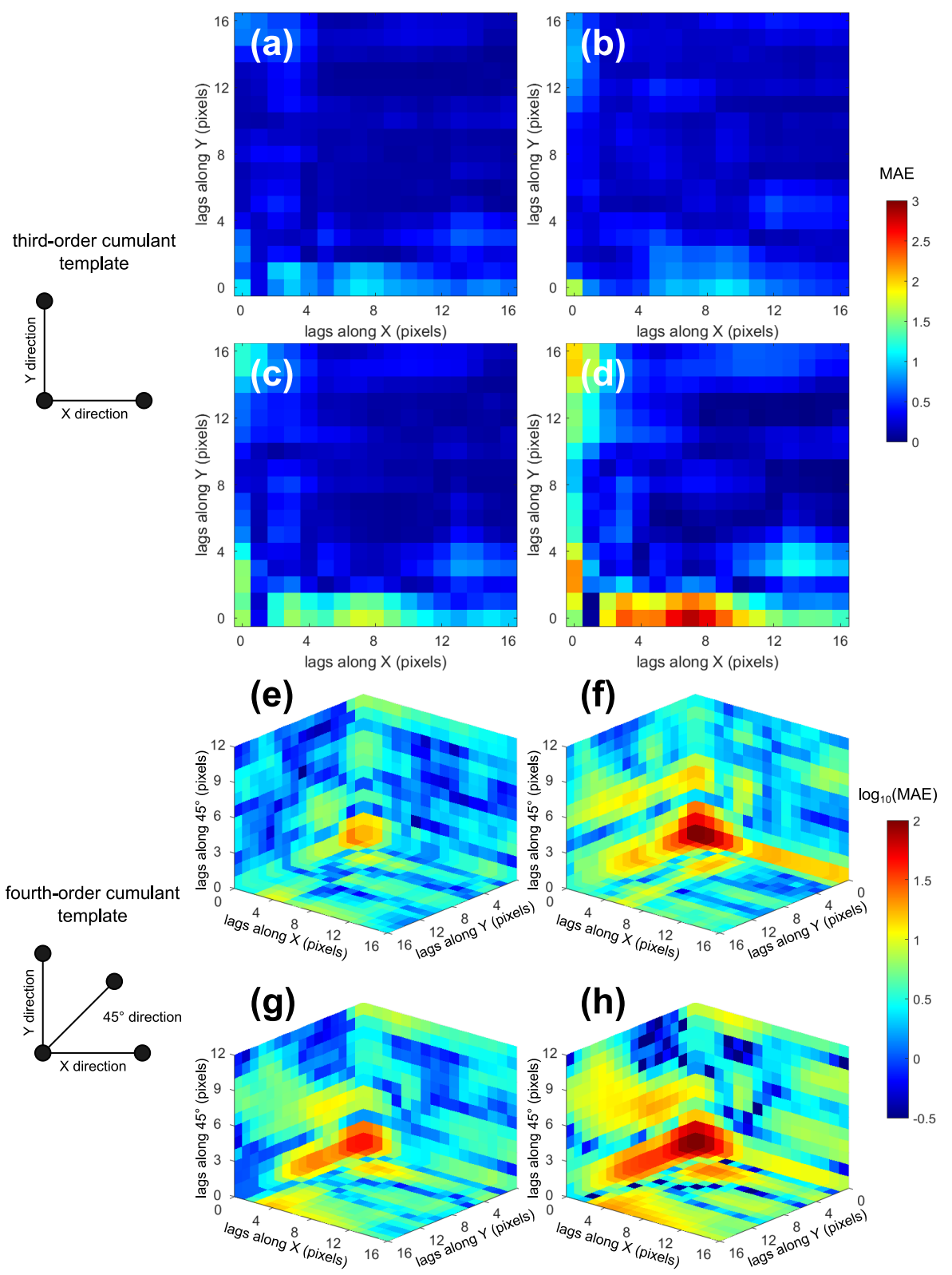

Fig. 22 MAE between simulated and reference third and fourth-order cumulant maps from the Jura Mountains example $(G=2)$. Top: third-order cumulant MAE maps for a proposed method, b area-to-point simulation, $\mathbf{c}$ direct sampling, and $\mathbf{d}$ bicubic interpolation. Colorbar unit is $\mathrm{m}^{3}$. Bottom: fourth-order cumulant MAE maps for $\mathbf{e}$ proposed method, $\mathbf{f}$ area-to-point simulation, $\mathbf{g}$ direct sampling, and $\mathbf{h}$ bicubic interpolation. Colorbar unit is $\mathrm{m}^{4}$. 

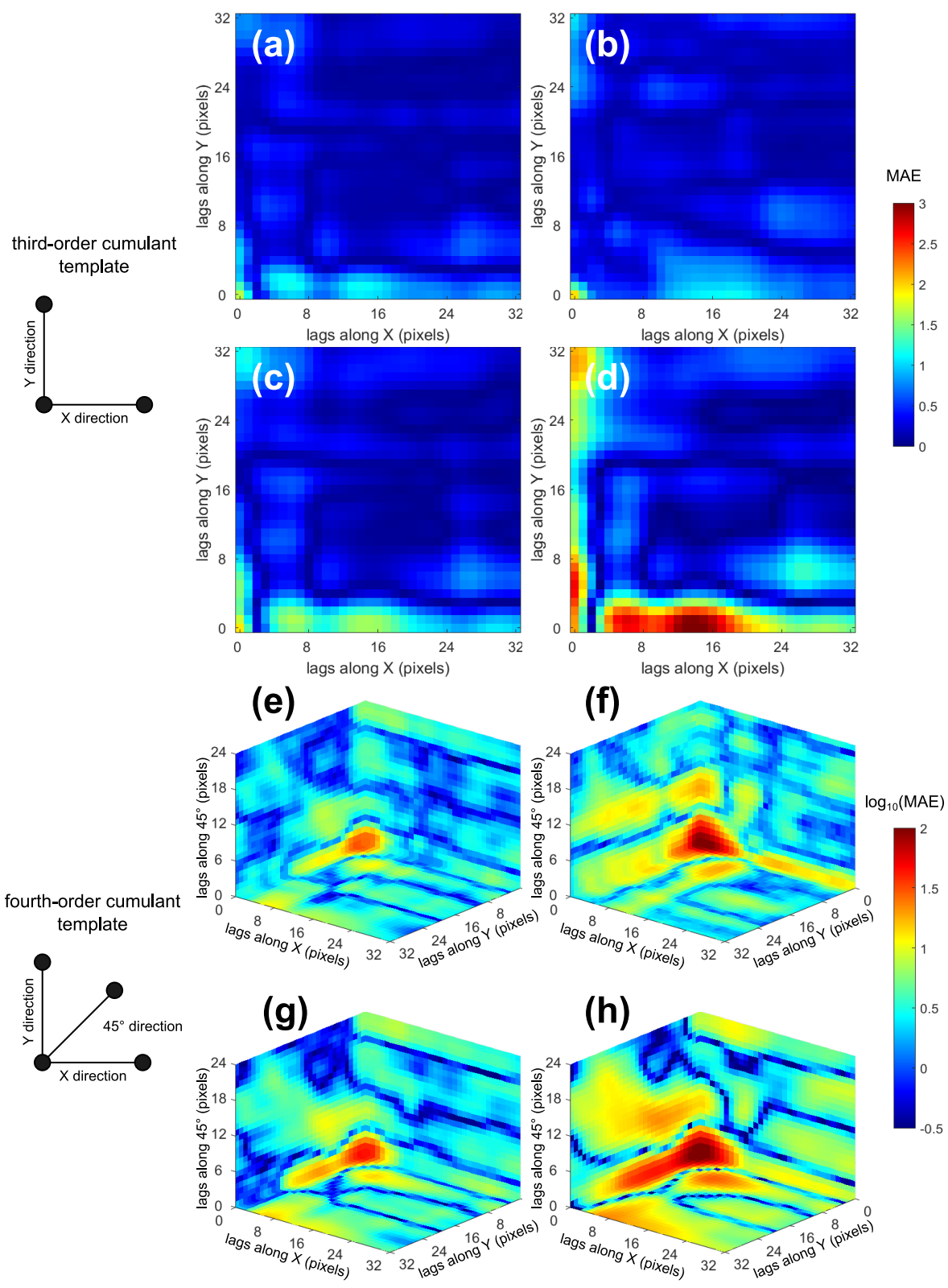

Fig. 23 MAE between simulated and reference third and fourth-order cumulant maps from the Jura Mountains example $(G=4)$. Top: third-order cumulant MAE maps for a proposed method, b area-to-point simulation, c direct sampling, and $\mathbf{d}$ bicubic interpolation. Colorbar unit is $\mathrm{m}^{3}$. Bottom: fourth-order cumulant MAE maps for $\mathbf{e}$ proposed method, $\mathbf{f}$ area-to-point simulation, $\mathbf{g}$ direct sampling, and $\mathbf{h}$ bicubic interpolation. Colorbar unit is $\mathrm{m}^{4}$. 